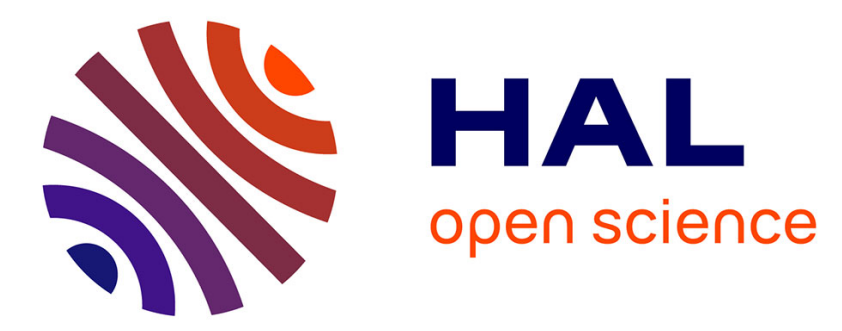

\title{
Spirobicyclic and Tetracyclic Pyrazolidinones: Syntheses and Properties
}

Fabien Perez, Thibault Leveille, Mathilde Bertolotti, Jean Rodriguez, Yoann Coquerel

\section{- To cite this version:}

Fabien Perez, Thibault Leveille, Mathilde Bertolotti, Jean Rodriguez, Yoann Coquerel. Spirobicyclic and Tetracyclic Pyrazolidinones: Syntheses and Properties. European Journal of Organic Chemistry, 2019, 2019 (35), pp.6034-6043. 10.1002/ejoc.201900989 . hal-02331699

\section{HAL Id: hal-02331699 \\ https://hal.science/hal-02331699}

Submitted on 24 Oct 2019

HAL is a multi-disciplinary open access archive for the deposit and dissemination of scientific research documents, whether they are published or not. The documents may come from teaching and research institutions in France or abroad, or from public or private research centers.
L'archive ouverte pluridisciplinaire HAL, est destinée au dépôt et à la diffusion de documents scientifiques de niveau recherche, publiés ou non, émanant des établissements d'enseignement et de recherche français ou étrangers, des laboratoires publics ou privés. 


\title{
Spirobicyclic and Tetracyclic Pyrazolidinones: Syntheses and Properties
}

\author{
Fabien Perez, ${ }^{[a]}$ Thibault Leveille, ${ }^{[a]}$ Mathilde Bertolotti, ${ }^{[a]}$ Jean Rodriguez, ${ }^{*[a]}$ and \\ Yoann Coquerel*[a]
}

Dedicated to the memory of Professor Dr. Dieter Enders

Abstract: The stereoselective syntheses of spirobicyclic and tetracyclic pyrazolidinones are reported based on a $(3+2)$ annulation between hydrazones and $\alpha$-oxoketenes. Some of these conformationally constrained molecules were resolved as enan- tiopure materials by HPLC techniques and evaluated as aminocatalysts for iminium activation in a model Diels-Alder cycloaddition.

\section{Introduction}

The use of small chiral and enantiopure organic molecules mimicking the activity of enzymes in accelerating enantioselective organic chemical transformations is now referred to as enantioselective organocatalysis. ${ }^{[1]}$ For instance, some organocatalysts embedding a basic nitrogen atom are amenable to the activation of enals through the temporary catalytic formation of the corresponding iminium ions to trigger, among others, Michael additions and/or Diels-Alder cycloadditions. ${ }^{[2]}$ Most often, the iminium activation of enals has been found efficient with the so-called Hayashi-Jørgensen and/or MacMillan's catalysts (Figure 1a), ${ }^{[2 a-2 d]}$ which usually induced good enantioselectivity. Despite their broad applicability, these catalysts are not ideal with somewhat improvable stereoselectivities (typically 90$95 \%$ ee, rarely $>98 \%$ ee) and kinetics (typically 10 mol-\% catalyst loading for hours to days). Some alternative catalysts have been proposed in order to overcome these limitations, notably based on the hydrazide moiety (Figure $1 \mathrm{~b}$ ). ${ }^{[3]}$ Herein, we report modular stereoselective syntheses of spirobicyclic and tetracyclic pyrazolidinones of type 1-3 in a few steps from readily available chemicals (Figure 1c), as well as the evaluation of their properties as catalysts for the iminium activation of enals in a model Diels-Alder cycloaddition. a) The most famous and used catalysts

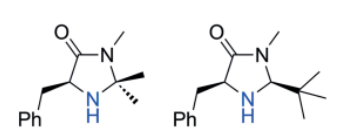

MacMillan, 2000 \& 2003

b) Alternative hydrazide-based catalysts

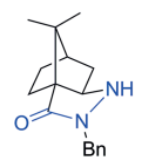

Ogilvie, 2005<smiles>[R]NS(=O)(=O)C1(CC)CCC2CCC1(C)C2</smiles>

$R=E t$, Lee, 2008 $\mathrm{R}=\mathrm{Bn}$, Langlois, 2008<smiles>CC(C)NN1C(=O)N(NC(C)C)C(c2ccccc2)C1c1ccccc1</smiles>

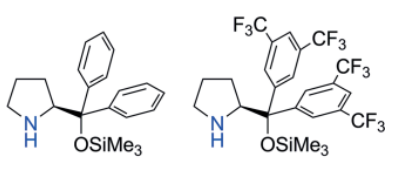

Hayashi-Jørgensen, 2005<smiles>CC(=O)CC(NNC(=O)[C@@H]1CCCN1C(=O)OC(C)(C)C)C(F)(F)F</smiles><smiles>O=C1CC(c2ccccc2)NN1C(=O)OCc1ccccc1</smiles><smiles>CC1CCC2C(C1)NN(C(=O)c1ccccc1)C2(C)C</smiles>

Bach, 2010<smiles>CC1CCC(C(C)C)C(OC(=O)N2CCCCC(c3ccccc3)N2)C1</smiles>

c) This work

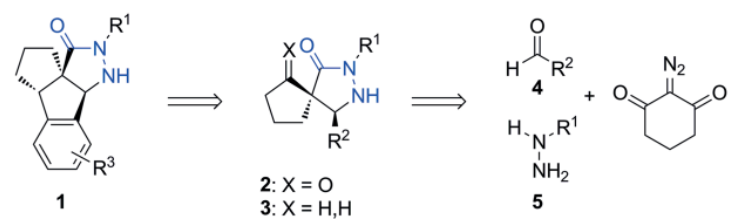

Figure 1. Aminocatalysts for the enantioselective iminium activation of enals and highlights of the present work.

\section{Results and Discussion}

CNRS, Centrale Marseille, ISM2, 13397 Marseille, France

E-mail: jean.rodriguez@univ-amu.fr yoann.coquerel@univ-amu.fr

http://ism2.univ-amu.fr/en-gb/user/2207 (JR)

http://ism2.univ-amu.fr/en-gb/user/2204 (YC)

We have earlier shown that $\alpha$-oxoketenes, generated in situ by a microwave-assisted Wolff rearrangement of 2-diazo-1,3-dicarbonyl compounds, ${ }^{[4]}$ are effective dipolarophiles in their reactions with azomethine imines in three-component processes, which allowed the expeditious stereoselective syntheses of 
some spirobicyclic pyrazolidinones such as $2 .{ }^{[5]}$ Originally, the required hydrazone intermediates, which upon heating undergo a 1,2-proton shift to give the corresponding reactive azomethine imines, were prepared in situ from carbonyl compounds 4 and hydrazines 5 in the presence of the required diazo compounds (three-component reaction). The formation of the hydrazones from the corresponding carbonyl compounds and hydrazines is accompanied by the formation of an equimolar amount of water, and the latter can concurrently react with $\alpha$-oxoketenes to give the corresponding unstable $\beta$ ketocarboxylic acids. For the complementary work herein, and in the interest of efficiency, we have modified the original multicomponent procedure for a consecutive protocol allowing for the elimination of undesired water after the formation of the hydrazone intermediates. In practice, as an illustrative example, a 1:1 mixture of benzaldehyde (4a) and phenyl hydrazine (5a) in anhydrous toluene was irradiated with microwaves at $140^{\circ} \mathrm{C}$ for 5 minutes, cooled down to $55^{\circ} \mathrm{C}$, concentrated and dried in vacuo. To the resulting material was added 2-diazo-1,3-cyclohexanedione and anhydrous toluene, and the mixture was irradiated with microwaves at $140{ }^{\circ} \mathrm{C}$ for 10 additional minutes, which afforded after purification $86 \%$ yield of the expected spiro product $2 \mathbf{a}$ and a trace amount of the uncyclized acylhydrazone 6 a (Table 1, entry 1). In comparison, only $57 \%$ yield was obtained for $\mathbf{2} \mathbf{a}$ using the multicomponent protocol. ${ }^{[5]}$ Using this method, we attempted the syntheses of the representa-

Table 1. Synthesis of spirobicyclic pyrazolidinones 2. $^{\text {[a] }}$

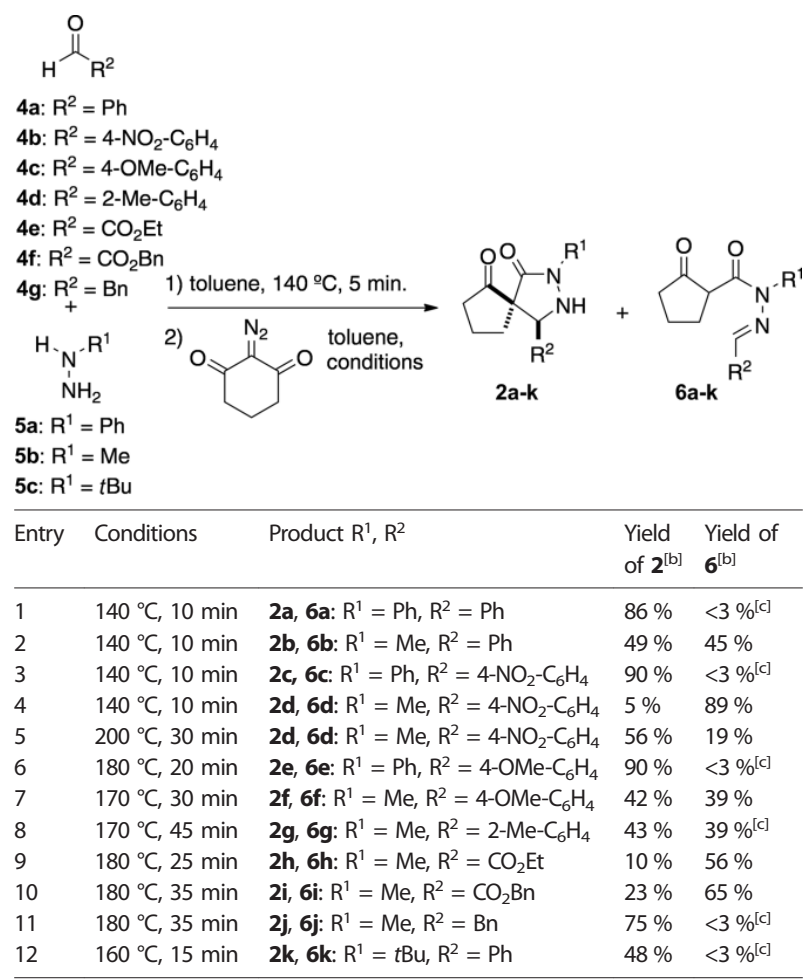

[a] All reactions were performed under microwave irradiation in sealed vessels. [b] Yields for the isolated product after purification unless otherwise noted. [c] Estimated by ${ }^{1} \mathrm{H}$ NMR analysis of the crude reaction mixture. tive spirobicyclic pyrazolidinones $\mathbf{2} \mathbf{b}-\mathbf{k}$. While reactions with phenyl and tert-butyl hydrazines (5a and $\mathbf{5 c}$, respectively) proceeded without event (entries 1, 3 and 12), the reactions with methyl hydrazine (5b) were complicated by the formation of the uncyclized acylhydrazones $\mathbf{6}$ in various proportions, formally resulting from the simple nucleophilic addition of the intermediate hydrazone to the intermediate $\alpha$-oxoketene. For instance, only $5 \%$ of $\mathbf{2 d}$ could be obtained together with $89 \%$ of $\mathbf{6 d}$ from a reaction performed at $140{ }^{\circ} \mathrm{C}$ for $10 \mathrm{~min}$ (entry 4). However, increasing the reaction temperature to $200{ }^{\circ} \mathrm{C}$ for 30 min allowed obtaining $\mathbf{2} \mathbf{d}$ as the major product in $56 \%$ yield (entry 5). In a separate experiment, the uncyclized acylhydrazone $\mathbf{6 h}$ could be converted into its spirobicyclic pyrazolidinone isomer $\mathbf{2 h}$ in $75 \%$ yield by heating at $190^{\circ} \mathrm{C}$ for 3 hours, showing that $\mathbf{6} \mathbf{h}$ is the kinetic isomer and $\mathbf{2} \mathbf{h}$ the thermodynamic one. Calculations of the free Gibbs energies of $\mathbf{6} \mathbf{h}$ and $\mathbf{2} \mathbf{h}$ by DFT methods indicated that $\mathbf{2} \mathbf{h}$ is indeed stabilized by ca. $37.4 \mathrm{~kJ} \mathrm{~mol}^{-1}$ relative to $\mathbf{6} \mathbf{h}$ (see the Supporting Information). The syntheses of derivatives $\mathbf{3}$ and $\mathbf{1}$ from $\mathbf{2}$ were devised through the intermediacy of the corresponding triflyl enol ethers 8 using catalytic palladium(0) chemistry. Thus, some selected spirobicyclic pyrazolidinones $\mathbf{2}$ were protected as the corresponding methyl or benzyl carbamates $\mathbf{7}$, which were then converted into the desired triflyl enol ethers $\mathbf{8}$ under standard conditions (Scheme 1). The relatively lower yields observed for the formation of the corresponding triflyl enol ethers $\mathbf{8 h}_{\mathbf{B n}}$ and $\mathbf{8}_{\mathbf{B}}$ from ketones $\mathbf{7 h}_{\mathbf{B n}}$ and $\mathbf{7} \mathbf{i}_{\mathbf{B n}}$ having $R^{2}$ ester substituents are probably due to the presence of a competing enolizable position in these substrates.

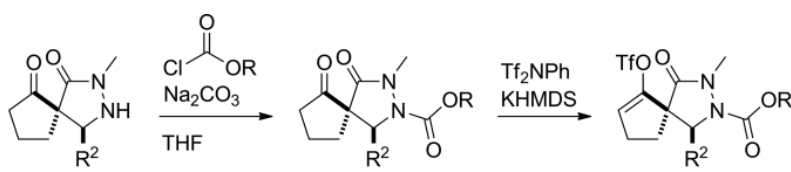

2a: $R^{2}=P h$

2d: $\mathrm{R}^{2}=4-\mathrm{NO}_{2}-\mathrm{C}_{6} \mathrm{H}_{4}$

2f: $\mathrm{R}^{2}=4-\mathrm{OMe}^{-} \mathrm{C}_{6} \mathrm{H}_{4}$

2g: $\mathrm{R}^{2}=2-\mathrm{Me}^{-} \mathrm{C}_{6} \mathrm{H}_{4}$

2h: $\mathrm{R}^{2}=\mathrm{CO}_{2} \mathrm{Et}$

2j: $R^{2}=B n$ 2i: $\mathrm{R}^{2}=\mathrm{CO}_{2} \mathrm{Bn}$
$7 \mathrm{a}_{\mathrm{Me}}: \mathrm{R}=\mathrm{Me}, 88 \%$

$7 \mathrm{a}_{\mathrm{Bn}}: \mathrm{R}=\mathrm{Bn}, 78 \%$

$7 \mathrm{~d}_{\mathrm{Me}}: \mathrm{R}=\mathrm{Me}, 99 \%$

$7 \mathrm{f}_{\mathrm{Me}}: \mathrm{R}=\mathrm{Me}, 99 \%$

$\mathbf{7 g}_{\mathrm{Me}}: \mathrm{R}=\mathrm{Me}, 99 \%$

$7 \mathrm{~h}_{\mathrm{Bn}}: \mathrm{R}=\mathrm{Bn}, 80 \%$

$7 \mathrm{i}_{\mathrm{Bn}}: \mathrm{R}=\mathrm{Bn}, 52 \%$

$7 \mathrm{j}_{\mathrm{Bn}}: \mathrm{R}=\mathrm{Bn}, 78 \%$
$8 a_{M e}: R=M e, 89 \%$

$8 a_{B n}: R=B n, 84 \%$

$8 \mathrm{~d}_{\mathrm{Me}}: \mathrm{R}=\mathrm{Me}, 71 \%$

$\mathbf{8 f}_{\mathrm{Bn}}: \mathrm{R}=\mathrm{Bn}, 73 \%$

$8 \mathrm{~g}_{\mathrm{Me}}: \mathrm{R}=\mathrm{Me}, 67 \%$

$8_{h_{B n}}: R=B n, 46 \%$

$8 i_{B n}: R=B n, 41 \%$

$8 \mathrm{j}_{\mathrm{Bn}}: \mathrm{R}=\mathrm{Bn}, 84 \%$
Scheme 1. Generation of triflyl enol ethers $\mathbf{8}$.

The vinyl triflates $\mathbf{8}_{\mathbf{B}}, \mathbf{8 i}_{\mathbf{B} \mathbf{n}}$ and $\mathbf{8}_{\mathbf{B} \mathbf{n}}$ were then subjected to palladium-catalyzed reduction conditions using some ammonium formate as the source of hydride, which afforded the expected reduced products $\mathbf{9}_{\mathbf{B}}, \mathbf{9 i}_{\mathbf{B n}}$ and $\mathbf{9}_{\mathbf{B n}}$, respectively (Scheme 2). Treatment of $\mathbf{9} \mathbf{h}_{\mathbf{B n}}$ and $\mathbf{9 j}_{\mathbf{B n}}$ with hydrogen gas in the presence of catalytic $\mathrm{Pd} / \mathrm{C}$ led to the concomitant hydrogenation of the double bond and cleavage of the benzyl carbamate to give the spirobicyclic pyrazolidinones $\mathbf{3 h}$ and $\mathbf{3} \mathbf{j}$, respectively. Significantly, $\mathbf{3} \mathbf{j}$ slowly oxidized to the corresponding spirobicyclic acylhydrazone when exposed to air or nondegassed solvents (a reaction similar to $\mathbf{3 b} \rightarrow \mathbf{1 2}$ in Scheme 3). The scenario was found different with the vinyl triflates 8 having an aromatic $R^{2}$ group. Indeed, the triflate and the aryl $R^{2}$ groups are in the same region of space for stereochemical reasons, and some competing palladium-catalyzed cyclization may 
occur through activation of the aromatic $\mathrm{C}-\mathrm{H}$ bond at $\mathrm{C} 2 .{ }^{[6]}$ In practice, only $\mathrm{C}-\mathrm{H}$ activation products were observed, even in the presence of formic acid, and excellent yields of the tetracyclic compounds $\mathbf{1 0}$ were achieved (Scheme 2). Methanolysis of the methyl carbamate $\mathbf{1 0 a _ { M e }}$ with $\mathrm{LiOH} / \mathrm{MeOH}$ was found slow and moderately selective, affording a modest $39 \%$ yield of the tetracyclic hydrazide $\mathbf{1 0 a}$. The concomitant diastereoselective hydrogenation/benzyl carbamate cleavage of $\mathbf{1 0 f}_{\mathrm{Bn}}$ afforded the tetracyclic pyrazolidinone 1f. The structures of $10 \mathbf{a}$ and the trifluoromethylsulfonate salt $\mathbf{1} \mathbf{f} \cdot \mathbf{C F}_{3} \mathbf{S O}_{3} \mathbf{H}$ were elucidated by monocrystal X-ray diffraction techniques, confirming the syntheses outcomes (Figure 2). ${ }^{[7]}$

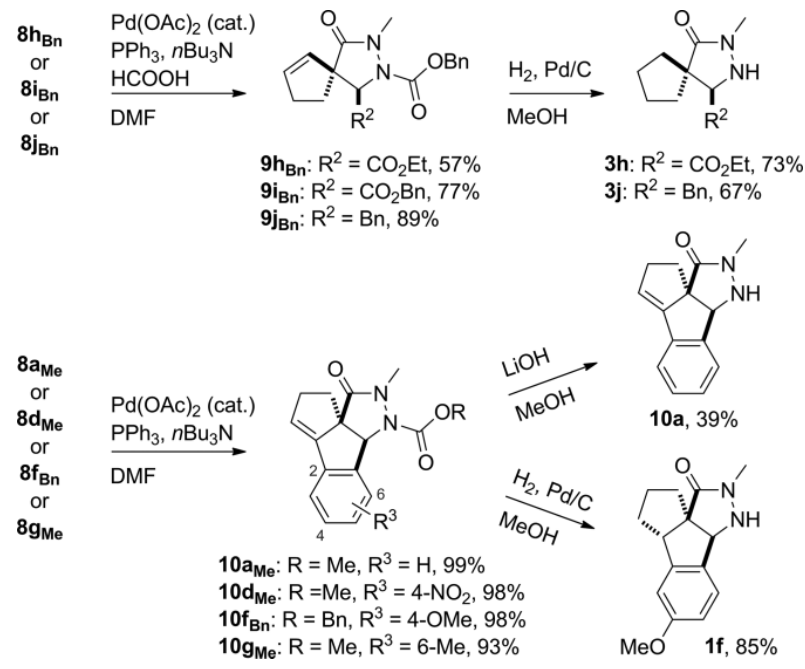

Scheme 2. Syntheses of the spirobicyclic pyrazolidinones $\mathbf{3} \mathbf{h}, \mathbf{j}$ and the tetracyclic pyrazolidinones $10 a, 1 f$.

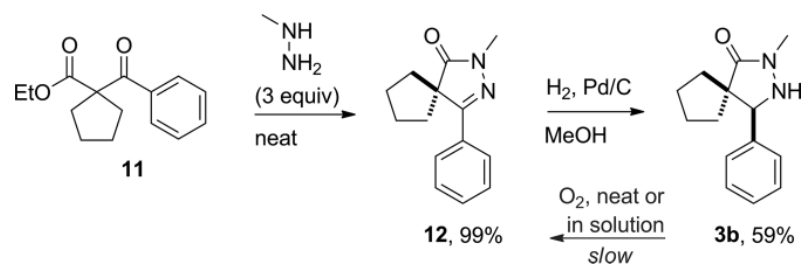

Scheme 3. Synthesis of the spirobicyclic pyrazolidinone $\mathbf{3 b}$.

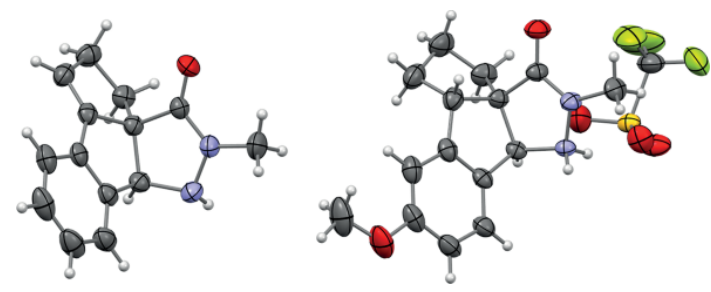

Figure 2. 3D representations of $\mathbf{1 0 a} \mathbf{a}_{\mathbf{M e}}$ (left) and $\mathbf{1} \mathbf{f} \cdot \mathbf{C F}_{3} \mathbf{S O}_{3} \mathbf{H}$ (right) obtained by monocrystal $\mathrm{X}$-ray diffraction analyses. Ellipsoids are draw at the $50 \%$ probability level and $\mathrm{H}$ atoms are draw as fixed-size spheres of $0.15 \AA$ radius. $^{[7]}$

An alternative synthetic approach was devised for the spirobicyclic pyrazolidinone $\mathbf{3 b}$ not available by the above sequence (Scheme 3). Cyclopentane 11, readily prepared by double $C$-alkylation of the corresponding $\beta$-ketoester with 1,4-dibromobutane, ${ }^{[8]}$ was treated with methyl hydrazine to give acylhydrazone 12, the hydrogenation of which afforded pyrazolidinone $\mathbf{3} \mathbf{b}$. Like its analog $\mathbf{3} \mathbf{j}$, pyrazolidinone $\mathbf{3} \mathbf{b}$ was found somewhat unstable in the presence of oxygen and slowly oxidized back to the acylhydrazone $\mathbf{1 2}$.

A motivation for this synthetic work was the identification of a competent catalyst for iminium activation in this series. Our efforts in this direction are summarized below. It was rapidly found that $\beta$-ketopyrazolidinones $\mathbf{2}$ were not suitable. For instance, $\mathbf{2 d}$ did not promote the Diels-Alder cycloaddition between cinnamaldehyde (13) and cyclopentadiene (14) in the absence of an acidic co-catalyst, while it was rapidly converted into the ring-opened acylhydrazone $\mathbf{6} \mathbf{d}$ in the presence of trifluoroacetic acid. We focused our efforts on pyrazolidinones $\mathbf{3 h}$, $\mathbf{3 j}$, $\mathbf{3} \mathbf{b}$ and $\mathbf{1 f}$. The stoichiometric formation of the iminium ion derived from hydrazide $\mathbf{3 h}$ and cinnamaldehyde (13) was quantitatively evaluated in the presence of weak to super acids (Figure 3). In all cases equilibrium was reached after a few hours at $25^{\circ} \mathrm{C}$. Benzoic acid, the weakest, left the substrates unchanged, and trifluoromethanesulfonic acid, the strongest, promoted a nearly quantitative formation of the (E)-configured iminium ion exclusively, as determined by NMR (the iminium ion derived from hydrazide $\mathbf{3} \mathbf{j}$ and $\mathbf{1 3}$ was also found (E)-configured, see the Supporting Information) and in full agreement with previous observations. ${ }^{[3 a, 3 c]}$
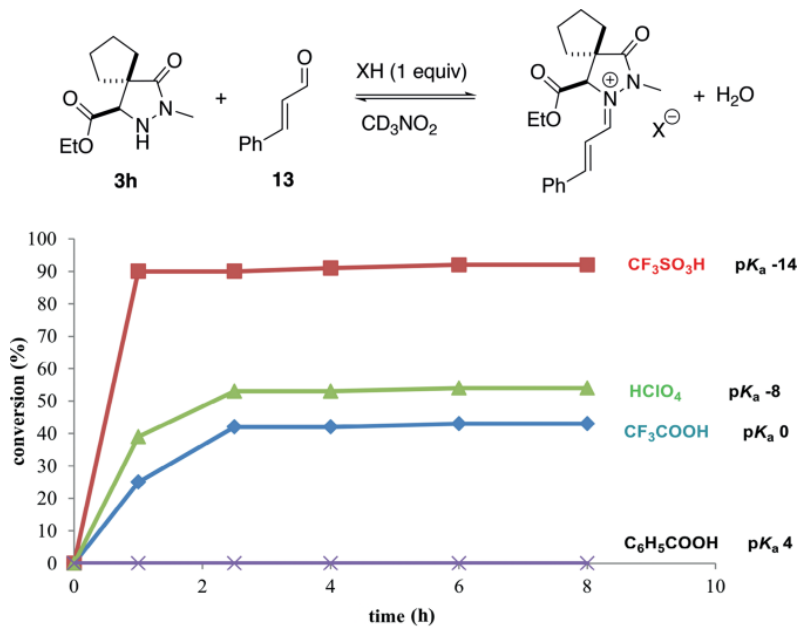

Figure 3. Formation of the iminium ion derived from pyrazolidinone $\mathbf{3 h}$ and cinnamaldehyde (13) at $25{ }^{\circ} \mathrm{C}$ in $d_{3}$-nitromethane. Conversions were determined by ${ }^{1} \mathrm{H}$ NMR.

The enantiomers of pyrazolidinones $\mathbf{3} \mathbf{h}, \mathbf{3} \mathbf{j}$ and $\mathbf{3} \mathbf{b}$ were resolved by semi-preparative HPLC on chiral stationary phases (see the Supporting Information) and their catalytic properties evaluated, together with those of racemic $\mathbf{1 f}$, in the model enantioselective (not for 1f) Diels-Alder cycloaddition between cinnamaldehyde (13) and cyclopentadiene (14). Our best, but rather modest, results are summarized in Scheme 4. Pyrazolidinone (+)-3h promoted the reaction at an acceptable rate but with virtually no stereoselectivity, while pyrazolidinone (+)-3j was found to induce some enantioselectivity, $71 \%$ ee at best 


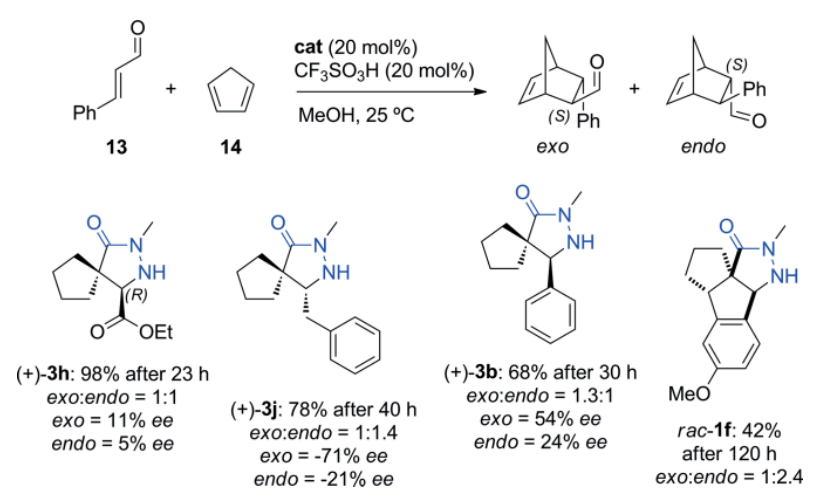

Scheme 4. Evaluation of the organocatalytic properties of $\mathbf{3 h}, \mathbf{3} \mathbf{j}, \mathbf{3} \mathbf{b}$ and $\mathbf{1 f}$ for the enantioselective (not for 1f) iminium activation of cinnamaldehyde (13) in its Diels-Alder cycloaddition with cyclopentadiene (14). The absolute configurations of $(+)-\mathbf{3 h}$ was confirmed as $(R)$ by VCD techniques, the depicted absolute configurations for $(+)-\mathbf{3} \mathbf{j}$ and $(+)-\mathbf{3} \mathbf{b}$ are arbitrary and unverified.

The absolute configuration of (+)-3h was unambiguously assigned as $(R)$ by vibrational circular dichroism spectroscopy (see the Supporting Information). The absolute configurations of the major enantiomers of the cycloadducts obtained with (+)-3h and $(+)-\mathbf{3} \mathbf{b}$ were determined as $(S)$ at the carbon atom bearing the aldehyde by comparison with the cycloadducts obtained with the MacMillan's catalysts of known enantio-induction, ${ }^{[2 a]}$ and pyrazolidinone (+)-3j induced opposite enantioselectivity. Overall, these stereochemical considerations are fully consistent with an approach of the cyclopentadiene (14) from the $\alpha$ face of the (E)-configured iminium ions derived from (+)-3h, in full agreement with earlier studies.

\section{Conclusions}

In summary, the $(3+2)$ annulation reaction between $\alpha$-oxoketenes and hydrazones was confirmed as a powerful synthetic approach to diverse, structurally complex, conformationally constrained, and yet stereodefined polycyclic pyrazolidinones including spirobicyclic, bis-spirotricyclic (previous work ${ }^{[5]}$ ), and tetracyclic derivatives obtained in just a few steps from simple chemicals. Notably, palladium-catalyzed cyclizations allowed the efficient construction of tetracyclic backbones through aromatic $\mathrm{C}-\mathrm{H}$ bond activation. The catalytic properties of some selected pyrazolidinones were evaluated for the enantioselective iminium activation of cinnamaldehyde (13) in a model archetypal Diels-Alder cycloaddition without significant improvement when compared to known systems.

\section{Experimental Section}

General Procedure for Pyrazolidinones 2 and Acylhydrazones 6: A solution of hydrazine and aldehyde in anhydrous toluene (ca.

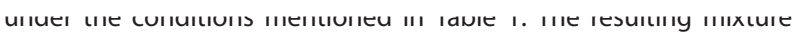
was cooled down to $55{ }^{\circ} \mathrm{C}$ by airflow, concentrated in vacuo and directly purified by flash chromatography. PE refers to petroleum ether (bp 40-60 ${ }^{\circ} \mathrm{C}$ ) and melting points are reported for amorphous material unless specified otherwise.

Pyrazolidinone 2a: Following the general procedure with benzaldehyde ( $366 \mu \mathrm{L}, 3.62 \mathrm{mmol})$, phenylhydrazine ( $359 \mu \mathrm{L}, 3.62 \mathrm{mmol}$ ) and 2-diazo-1,3-cyclohexanedione (500 mg, $3.62 \mathrm{mmol}$ ) in $9 \mathrm{~mL}$ of anhydrous toluene at $140{ }^{\circ} \mathrm{C}$ for $10 \mathrm{~min}$, and using EtOAc/PE, 1:9 as eluent for the flash chromatography, 2 a was obtained as a white solid (953 mg, $86 \%)$. The ${ }^{1} \mathrm{H}$ NMR analysis of the crude material showed that no more than $3 \%$ of acylhydrazone $\mathbf{6 a}$ (not isolated) was formed. $\boldsymbol{R}_{\mathbf{f}}$ (EtOAc/PE, 1:9) $=0.36 .{ }^{1} \mathrm{H}$ NMR $(300 \mathrm{MHz}, \delta \mathrm{ppm} /$ $\mathrm{CDCl}_{3}$ ): 7.92 (dd, $\left.J=1.2,8.8 \mathrm{~Hz}, 2 \mathrm{H}\right), 7.44-7.31(\mathrm{~m}, 7 \mathrm{H}), 7.15$ (pseudo $\mathrm{t}, J=7.4 \mathrm{~Hz}, 1 \mathrm{H}), 5.60(\mathrm{~d}, J=12.8 \mathrm{~Hz}, 1 \mathrm{H}), 4.66(\mathrm{~d}, J=12.9 \mathrm{~Hz}, 1 \mathrm{H})$, $2.73(\mathrm{ddd}, J=13.5,8.2,8.2 \mathrm{~Hz}, 1 \mathrm{H}), 2.27-2.09(\mathrm{~m}, 2 \mathrm{H}), 2.07-1.95(\mathrm{~m}$, $1 \mathrm{H}), 1.89-1.75(\mathrm{~m}, 1 \mathrm{H}), 1.64-1.47(\mathrm{~m}, 1 \mathrm{H})$. These data are in agreement with previously reported data for this compound. ${ }^{[5]}$

Pyrazolidinone $\mathbf{2 b}$ and Acylhydrazone $\mathbf{6 b}$ : Following the general procedure with benzaldehyde ( $92 \mu \mathrm{L}, 0.90 \mathrm{mmol})$, methylhydrazine (54 $\mu \mathrm{L}, 1.03 \mathrm{mmol}$ ) and 2-diazo-1,3-cyclohexanedione (125 mg, $0.90 \mathrm{mmol}$ ) in $3 \mathrm{~mL}$ of anhydrous toluene at $140{ }^{\circ} \mathrm{C}$ for $10 \mathrm{~min}$, and using AcOEt/PE, 4:6 as eluent for the flash chromatography, $\mathbf{6} \mathbf{b}$ and $\mathbf{2 b}$ were obtained, in that order, as a white solids $(\mathbf{6 b}: 100 \mathrm{mg}$, 45 \%; 2 b:127 mg, $49 \%$ ). For $6 \mathbf{b}: R_{\mathrm{f}}(\mathrm{AcOEt} / \mathrm{PE}, 4: 6)=0.60$. Mp 109.4$111.4{ }^{\circ} \mathrm{C}$. HRMS $\left(\mathrm{ESI}^{+}\right):[\mathrm{M}+\mathrm{H}]^{+}$calcd. for $\mathrm{C}_{14} \mathrm{H}_{17} \mathrm{~N}_{2} \mathrm{O}_{2}{ }^{+}$245.1285, found 245.1287. ${ }^{1} \mathrm{H}$ NMR (300 MHz, $\left.\delta \mathrm{ppm} / \mathrm{CDCl}_{3}\right)$ : $7.74(\mathrm{~s}, 1 \mathrm{H}), 7.65-$ $7.61(\mathrm{~m}, 2 \mathrm{H}), 7.44-7.35(\mathrm{~m}, 2 \mathrm{H}), 4.44(\mathrm{dd}, J=9.7,9.7 \mathrm{~Hz}, 1 \mathrm{H}), 3.42$ $(\mathrm{s}, 3 \mathrm{H}), 2.45-2.33(\mathrm{~m}, 4 \mathrm{H}), 2.28-2.13(\mathrm{~m}, 1 \mathrm{H}), 2.04-1.84(\mathrm{~m}, 1 \mathrm{H}) .{ }^{13} \mathrm{C}$ NMR (75 MHz, $\left.\delta \mathrm{ppm} / \mathrm{CDCl}_{3}\right): 214.0(\mathrm{C}), 171.6(\mathrm{C}), 140.0(\mathrm{CH}), 134.7$ (C), $129.9(\mathrm{CH}), 128.9(2 \mathrm{CH}), 127.3(2 \mathrm{CH}), 53.1(\mathrm{CH}), 38.6\left(\mathrm{CH}_{2}\right), 28.4$ $\left(\mathrm{CH}_{3}\right), 28.1\left(\mathrm{CH}_{2}\right), 21.4\left(\mathrm{CH}_{2}\right)$. For 2b: $R_{\mathrm{f}}(\mathrm{AcOEt} / \mathrm{PE}, 4: 6)=0.12 . \mathrm{Mp}$ 101.6-103.6 ${ }^{\circ} \mathrm{C}$. HRMS $\left(E S I^{+}\right)$: $[\mathrm{M}+\mathrm{H}]^{+}$calcd. for $\mathrm{C}_{14} \mathrm{H}_{17} \mathrm{~N}_{2} \mathrm{O}_{2}{ }^{+}$ 245.1285, found 245.1287. ${ }^{1} \mathrm{H}$ NMR (300 MHz, $\delta \mathrm{ppm} / \mathrm{CDCl}_{3}$ ): 7.39$7.33(\mathrm{~m}, 3 \mathrm{H}), 7.30-7.26(\mathrm{~m}, 2 \mathrm{H}), 5.22(\mathrm{~d}, J=12.0 \mathrm{~Hz}, 1 \mathrm{H}), 4.52(\mathrm{~d}, J=$ $12.0 \mathrm{~Hz}, 1 \mathrm{H}), 3.21(\mathrm{~s}, 3 \mathrm{H}), 2.63(\mathrm{ddd}, J=13.8,8.2,8.2 \mathrm{~Hz}, 1 \mathrm{H}), 2.21-$ $1.88(\mathrm{~m}, 4 \mathrm{H}), 1.73(\mathrm{ddd}, J=13.7,8.7,5.3 \mathrm{~Hz}, 1 \mathrm{H}), 1.49(\mathrm{~m}, 1 \mathrm{H}) .{ }^{13} \mathrm{C}$ NMR (75 MHz, $\left.\delta p p m / \mathrm{CDCl}_{3}\right): 216.2(\mathrm{C}), 171.1(\mathrm{C}), 133.4(\mathrm{C}), 129.3$ (C), $129.1(2 \mathrm{CH}), 127.5(2 \mathrm{CH}), 70.7(\mathrm{CH}), 63.7(\mathrm{C}), 39.2\left(\mathrm{CH}_{2}\right), 32.0$ $\left(\mathrm{CH}_{3}\right), 30.2\left(\mathrm{CH}_{2}\right), 19.8\left(\mathrm{CH}_{2}\right)$.

Pyrazolidinone 2c: Following the general procedure with $p$-nitrobenzaldehyde (138 mg, $0.90 \mathrm{mmol})$, phenylhydrazine $(91 \mu \mathrm{L}$, $0.90 \mathrm{mmol}$ ) and 2-diazo-1,3-cyclohexanedione (125 mg, $0.90 \mathrm{mmol}$ ) in $3 \mathrm{~mL}$ of anhydrous toluene at $140{ }^{\circ} \mathrm{C}$ for $10 \mathrm{~min}$, and using EtOAC/ $\mathrm{PE}, 3: 7$ as eluent for the flash chromatography, $\mathbf{2 c}$ was obtained as a red solid ( $286 \mathrm{mg}, 90 \%)$. The ${ }^{1} \mathrm{H}$ NMR analysis of the crude material showed that no more than $3 \%$ of acylhydrazone $6 \mathrm{c}$ (not isolated) was formed. $R_{\mathrm{f}}$ (AcOEt/PE, 3:7) $=0.28$. Mp: 157.4-159.4 ${ }^{\circ} \mathrm{C}$. HRMS $\left(E S I^{+}\right):[M+H]^{+}$calcd. for $\mathrm{C}_{19} \mathrm{H}_{18} \mathrm{~N}_{3} \mathrm{O}_{4}{ }^{+} 352.1292$, found 352.1291. ${ }^{1} \mathrm{H}$ NMR: $\left(300 \mathrm{MHz}, \delta \mathrm{ppm} / \mathrm{CDCl}_{3}\right): 8.26(\mathrm{~d}, J=8.7 \mathrm{~Hz}, 2 \mathrm{H})$, $7.89(\mathrm{~d}, J=7.9 \mathrm{~Hz}, 2 \mathrm{H}), 7.54(\mathrm{~d}, J=8.7 \mathrm{~Hz}, 2 \mathrm{H}), 7.39(\mathrm{dd}, J=7.9$, $7.9 \mathrm{~Hz}, 2 \mathrm{H}), 7.17(\mathrm{dd}, J=7.4 .7 .4 \mathrm{~Hz}, 1 \mathrm{H}), 5.68(\mathrm{~d}, J=12.7 \mathrm{~Hz}, 1 \mathrm{H})$, $4.80(\mathrm{~d}, J=12.7 \mathrm{~Hz}, 1 \mathrm{H}), 2.86-2.71(\mathrm{~m}, 1 \mathrm{H}), 2.30$ (ddd, $J=13.1,7.7$, $7.6 \mathrm{~Hz}, 1 \mathrm{H}), 2.22-2.08(\mathrm{~m}, 2 \mathrm{H}), 1.95-1.80(\mathrm{~m}, 1 \mathrm{H}), 1.71-1.56(\mathrm{~m}, 1 \mathrm{H})$. ${ }^{13} \mathrm{C}$ NMR (300 MHz, $\left.\delta \mathrm{ppm} / \mathrm{CDCl}_{3}\right): 215.3$ (C), 169.2 (C), $148.4(\mathrm{C})$, $140.6(\mathrm{C}), 138.5(\mathrm{C}), 129.0(2 \mathrm{CH}), 128.6(2 \mathrm{CH}), 125.2(\mathrm{CH}), 124.2(2 \mathrm{CH})$, $118.8(2 \mathrm{CH}), 68.4(\mathrm{CH}), 65.2(\mathrm{C}), 39.5\left(\mathrm{CH}_{2}\right), 30.3\left(\mathrm{CH}_{2}\right), 19.9\left(\mathrm{CH}_{2}\right)$. 
Pyrazolidinone 2d and Acylhydrazone 6d: Following the general procedure with $p$-nitro-benzaldehyde $(138 \mathrm{mg}, 0.90 \mathrm{mmol})$, methylhydrazine $(54 \mu \mathrm{L}, 1.03 \mathrm{mmol})$ and 2-diazo-1,3-cyclohexanedione (125 mg, $0.90 \mathrm{mmol}$ ) in $3 \mathrm{~mL}$ of anhydrous toluene at $140{ }^{\circ} \mathrm{C}$ for $10 \mathrm{~min}$, and using AcOEt/PE, 3:7 then 4:6 as eluent for the flash chromatography, $\mathbf{6} \mathbf{d}$ and $\mathbf{2 d}$ were obtained, in that order, as orange and yellow solids, respectively, (6d: $222 \mathrm{mg}, 89 \%$; $\mathbf{2 d : 1 2 ~ m g , ~} 5 \%$ ). A similar reaction performed at $200^{\circ} \mathrm{C}$ for 30 min afforded the same products $\mathbf{6 d}$ and $\mathbf{2 d}$ in $19 \%$ and $56 \%$ yield, respectively. For $\mathbf{6 d :} R_{\mathrm{f}}$ (AcOEt/PE, 3:7) $=0.47$. HRMS $\left(E S I^{+}\right):[\mathrm{M}+\mathrm{H}]^{+}$calcd. for $\mathrm{C}_{14} \mathrm{H}_{16} \mathrm{~N}_{3} \mathrm{O}_{4}{ }^{+}$ 290.1135, found 290.1135. Mp 166.4-168.4 ${ }^{\circ} \mathrm{C}$. ${ }^{1} \mathrm{H}$ NMR $(300 \mathrm{MHz}$, $\left.\delta \mathrm{ppm} / \mathrm{C}_{6} \mathrm{D}_{6}\right): 7.85(\mathrm{~d}, J=8.8 \mathrm{~Hz}, 2 \mathrm{H}), 7.11(\mathrm{~d}, J=8.8 \mathrm{~Hz}, 2 \mathrm{H}), 6.75$ $(\mathrm{s}, 1 \mathrm{H}), 4.31$ (dd, $J=10.4,8.8 \mathrm{~Hz}, 1 \mathrm{H}), 2.77(\mathrm{~s}, 3 \mathrm{H}), 2.37$ (dddd, $J=$ 12.7, 10.4, 10.4, $6.9 \mathrm{~Hz}, 1 \mathrm{H}), 1.99-1.83(\mathrm{~m}, 3 \mathrm{H}), 1.74-1.59(\mathrm{~m}, 1 \mathrm{H})$, $1.42-1.25(\mathrm{~m}, 1 \mathrm{H}) .{ }^{13} \mathrm{C}$ NMR $\left(75 \mathrm{MHz}, \delta \mathrm{ppm} / \mathrm{CDCl}_{3}\right): 213.5(\mathrm{C}), 171.5$ (C), $140.7(\mathrm{C}), 136.9(\mathrm{CH}), 127.7(2 \mathrm{CH}), 124.2,(2 \mathrm{CH}), 110.1(\mathrm{C}), 52.8$ $(\mathrm{CH}), 38.6\left(\mathrm{CH}_{2}\right), 28.7\left(\mathrm{CH}_{3}\right), 27.8\left(\mathrm{CH}_{2}\right), 21.2\left(\mathrm{CH}_{2}\right)$. For $2 \mathrm{~d}: R_{\mathrm{f}}(\mathrm{AcOEt} /$ $\left.\mathrm{CH}_{2} \mathrm{Cl}_{2}, 4: 6\right)=0.25$. Mp 156.0-157.0 ${ }^{\circ} \mathrm{C}$. HRMS $\left(\mathrm{ESI} \mathrm{H}^{+}\right):[\mathrm{M}+\mathrm{H}]^{+}$calcd. for $\mathrm{C}_{14} \mathrm{H}_{16} \mathrm{~N}_{3} \mathrm{O}_{4}{ }^{+}$290.1135, found 290.1137. ${ }^{1} \mathrm{H}$ NMR (300 MHz, $\delta$ ppm/ $\left.\mathrm{CDCl}_{3}\right): 8.23(\mathrm{~d}, J=8.6 \mathrm{~Hz}, 2 \mathrm{H}), 7.48(\mathrm{~d}, J=8.6 \mathrm{~Hz}, 2 \mathrm{H}), 5.28$ $(\mathrm{d}, J=12.3 \mathrm{~Hz}, 1 \mathrm{H}), 4.66(\mathrm{~d}, J=12.3 \mathrm{~Hz}, 1 \mathrm{H}), 3.21(\mathrm{~s}, 3 \mathrm{H}), 2.77-2.60$ $(\mathrm{m}, 1 \mathrm{H}), 2.31-2.15(\mathrm{~m}, 1 \mathrm{H}), 2.18-1.99(\mathrm{~m}, 2 \mathrm{H}), 1.88-1.71(\mathrm{~m}, 1 \mathrm{H})$, $1.68-1.47(\mathrm{~m}, 1 \mathrm{H}) .{ }^{13} \mathrm{C}$ NMR $\left(75 \mathrm{MHz}, \delta \mathrm{ppm} / \mathrm{CDCl}_{3}\right): 215.6(\mathrm{C}), 170.2$ (C), $148.3(\mathrm{C}), 140.9(\mathrm{C}), 128.5(2 \mathrm{CH}), 124.1(2 \mathrm{CH}), 69.2(\mathrm{CH}), 63.5(\mathrm{C})$, 39.4 $\left(\mathrm{CH}_{2}\right), 32.1\left(\mathrm{CH}_{2}\right), 30.3\left(\mathrm{CH}_{3}\right), 19.9\left(\mathrm{CH}_{2}\right)$.

Pyrazolidinone 2e: Following the general procedure with $p$-methoxy-benzaldehyde $(440 \mu \mathrm{L}, 3.62 \mathrm{mmol})$, phenylhydrazine $(359 \mu \mathrm{L}$, $3.62 \mathrm{mmol}$ ) and 2-diazo-1,3-cyclohexanedione (500 mg, $3.62 \mathrm{mmol}$ ) in $9 \mathrm{~mL}$ of anhydrous toluene at $180^{\circ} \mathrm{C}$ for $20 \mathrm{~min}$, and using EtOAc/ $\mathrm{PE}, 2: 8$ as eluent for the flash chromatography, $2 \mathbf{e}$ was obtained as an orange solid (1095 mg, $90 \%)$. The ${ }^{1} \mathrm{H}$ NMR analysis of the crude material showed that no more than $3 \%$ of acylhydrazone $\mathbf{6 e}$ (not isolated) was formed. $\boldsymbol{R}_{\mathbf{f}}(\mathrm{EtOAC} / \mathrm{PE}, 2: 8)=0.19$. $\mathrm{Mp} 160.9-162.9^{\circ} \mathrm{C}$. HRMS $\left(\right.$ ESI $\left.^{+}\right)$: $[\mathrm{M}+\mathrm{H}]^{+}$calcd. for $\mathrm{C}_{20} \mathrm{H}_{21} \mathrm{~N}_{2} \mathrm{O}_{3}{ }^{+} 337.1547$, found 337.1544. ${ }^{1} \mathrm{H}$ NMR $\left(300 \mathrm{MHz}, \delta \mathrm{ppm} / \mathrm{CDCl}_{3}\right): 7.95-7.87(\mathrm{~m}, 2 \mathrm{H}), 7.37$ (dd, $J=8.7,7.3 \mathrm{~Hz}, 2 \mathrm{H}), 7.28-7.24(\mathrm{~m}, 2 \mathrm{H}), 7.19-7.10(\mathrm{~m}, 1 \mathrm{H}), 6.91$ $(\mathrm{d}, J=8.7 \mathrm{~Hz}, 2 \mathrm{H}), 5.50(\mathrm{~d}, J=12.8 \mathrm{~Hz}, 1 \mathrm{H}), 4.60(\mathrm{~d}, J=12.7 \mathrm{~Hz}, 1 \mathrm{H})$, $3.82(\mathrm{~s}, 3 \mathrm{H}), 2.71$ (ddd, $J=13.8,8.1,8.1 \mathrm{~Hz}, 1 \mathrm{H}), 2.29-1.94(\mathrm{~m}, 3 \mathrm{H})$, 1.90-1.77 (m, 1H), 1.64-1.49 (m, 1H). ${ }^{13} \mathrm{C}$ NMR $(75 \mathrm{MHz}, \delta \mathrm{ppm} /$ $\left(\mathrm{CDCl}_{3}\right): 216.0$ (C), 170.1 (C), $160.3(\mathrm{C}), 138.9(\mathrm{C}), 128.9(2 \mathrm{CH}), 128.8$ $(2 \mathrm{CH}), 125.1(\mathrm{C}), 124.8(\mathrm{CH}), 118.8(2 \mathrm{CH}), 114.6(2 \mathrm{CH}), 69.4(\mathrm{CH}), 65.5$ (C), $55.4\left(\mathrm{CH}_{3}\right), 39.4\left(\mathrm{CH}_{2}\right), 30.3\left(\mathrm{CH}_{2}\right), 19.8\left(\mathrm{CH}_{2}\right)$.

Pyrazolidinone $\mathbf{2 f}$ and Acylhydrazone 6f: Following the general procedure with $p$-methoxy-benzaldehyde $(112 \mu \mathrm{L}, 0.90 \mathrm{mmol})$, methylhydrazine $(54 \mu \mathrm{L}, 1.03 \mathrm{mmol})$ and 2-diazo-1,3-cyclohexanedione $(125 \mathrm{mg}, 0.90 \mathrm{mmol})$ in $3 \mathrm{~mL}$ of anhydrous toluene at $170{ }^{\circ} \mathrm{C}$ for $30 \mathrm{~min}$, and using AcOEt/PE, 4:6 as eluent for the flash chromatography, $\mathbf{6} \mathbf{f}$ and $\mathbf{2} \mathbf{f}$ were obtained, in that order, as a colorless oil and a white solid, respectively, (6f: $97 \mathrm{mg}, 39 \%$; 2f:104 mg, $42 \%$ ). For 6f: $R_{\mathrm{f}}(\mathrm{AcOEt} / \mathrm{PE}, 4: 6)=0.30$. HRMS $\left(\mathrm{ESI} \mathrm{I}^{+}\right):[\mathrm{M}+\mathrm{H}]^{+}$calcd. for $\mathrm{C}_{15} \mathrm{H}_{19} \mathrm{~N}_{2} \mathrm{O}_{3}{ }^{+} 275.1390$, found 275.1389 . ' $\mathrm{H}$ NMR (300 MHz, $\delta \mathrm{ppm} /$ $\left.\mathrm{CDCl}_{3}\right): 7.68(\mathrm{~s}, 1 \mathrm{H}), 7.54(\mathrm{~d}, J=8.5 \mathrm{~Hz}, 2 \mathrm{H}), 6.90(\mathrm{~d}, J=8.5 \mathrm{~Hz}, 2 \mathrm{H})$, $4.39(\mathrm{dd}, J=9.6 .9 .6 \mathrm{~Hz}, 1 \mathrm{H}), 3.81(\mathrm{~s}, 3 \mathrm{H}), 3.36(\mathrm{~s}, 3 \mathrm{H}), 2.42-2.30(\mathrm{~m}$ $4 \mathrm{H}), 2.25-2.10(\mathrm{~m}, 1 \mathrm{H}), 2.03-1.80(\mathrm{~m}, 1 \mathrm{H}) .{ }^{13} \mathrm{C}$ NMR $(75 \mathrm{MHz}, \delta \mathrm{ppm} /$ $\left.\mathrm{CDCl}_{3}\right)$ : $214.1(\mathrm{C}), 171.4(\mathrm{C}), 161.1(\mathrm{C}), 139.8(\mathrm{CH}), 128.7(2 \mathrm{CH}), 127.4$ (C), $114.3(2 \mathrm{CH}), 55.4\left(\mathrm{CH}_{3}\right), 53.1(\mathrm{CH}), 38.5\left(\mathrm{CH}_{2}\right), 28.2\left(\mathrm{CH}_{3}\right), 28.1$ $\left(\mathrm{CH}_{2}\right), 21.3\left(\mathrm{CH}_{2}\right)$. For 2f: $R_{\mathrm{f}}(\mathrm{AcOEt} / \mathrm{PE}, 4: 6)=0.10$. HRMS $\left.(\mathrm{ESI})^{+}\right)$: $[\mathrm{M}+\mathrm{H}]^{+}$calcd. for $\mathrm{C}_{15} \mathrm{H}_{19} \mathrm{~N}_{2} \mathrm{O}_{3}{ }^{+} 275.1390$, found 275.1390 . Mp 131.4-133.4 ${ }^{\circ} \mathrm{C}$. ${ }^{1} \mathrm{H}$ NMR $\left(300 \mathrm{MHz}, \delta \mathrm{ppm} / \mathrm{CDCl}_{3}\right): 7.20(\mathrm{~d}, J=8.7 \mathrm{~Hz}$, $2 \mathrm{H}), 6.87(\mathrm{~d}, J=8.7 \mathrm{~Hz}, 2 \mathrm{H}), 5.14(\mathrm{~s}, 1 \mathrm{H}), 4.45(\mathrm{~s}, 1 \mathrm{H}), 3.80(\mathrm{~s}, 3 \mathrm{H})$, $3.20(\mathrm{~s}, 3 \mathrm{H}), 2.61(\mathrm{~d}, J=3.1 \mathrm{~Hz}, 1 \mathrm{H}), 2.13(\mathrm{~d}, J=1.5 \mathrm{~Hz}, 1 \mathrm{H}), 1.97(\mathrm{~s}$, 2H), $1.79(\mathrm{~s}, 1 \mathrm{H}), 1.50(\mathrm{~s}, 1 \mathrm{H}) .{ }^{13} \mathrm{C} \mathrm{NMR}\left(75 \mathrm{MHz}, \delta \mathrm{ppm} / \mathrm{CDCl}_{3}\right): 216.4$ (C), 171.0 (C), $160.2(\mathrm{C}), 128.7(2 \mathrm{CH}), 125.3(\mathrm{C}), 114.4(2 \mathrm{CH}), 70.3$
$(\mathrm{CH}), 63.7(\mathrm{C}), 55.4\left(\mathrm{CH}_{3}\right), 39.2\left(\mathrm{CH}_{2}\right), 31.9\left(\mathrm{CH}_{3}\right), 30.2\left(\mathrm{CH}_{2}\right), 19.8$ $\left(\mathrm{CH}_{2}\right)$.

Pyrazolidinone 2g: Following the general procedure with 2methyl-benzaldehyde $(420 \mu \mathrm{L}, 3.62 \mathrm{mmol})$, methylhydrazine $(213 \mu \mathrm{L}, 4.07 \mathrm{mmol})$ and 2-diazo-1,3-cyclohexanedione $(500 \mathrm{mg}$, $3.62 \mathrm{mmol}$ ) in $12 \mathrm{~mL}$ of anhydrous toluene at $170{ }^{\circ} \mathrm{C}$ for $45 \mathrm{~min}$, and using EtOAc/PE, 4:6 as eluent for the flash chromatography, $\mathbf{2 g}$ was obtained as a yellow solid (643 mg, $43 \%)$. The ${ }^{1} \mathrm{H}$ NMR analysis of the crude material evidenced the formation of ca. $39 \%$ of acylhydrazone $\mathbf{6 g}$ (not isolated). $R_{\mathrm{f}}(\mathrm{AcOEt} / \mathrm{PE}, 4: 6)=0.12$. Mp 164.6166.6 ${ }^{\circ} \mathrm{C}$. HRMS $\left(E S I^{+}\right):[\mathrm{M}+\mathrm{H}]^{+}$calcd. for $\mathrm{C}_{15} \mathrm{H}_{19} \mathrm{~N}_{2} \mathrm{O}_{2}{ }^{+}$259.1441, found 259.1440. ${ }^{1} \mathrm{H}$ NMR $\left(300 \mathrm{MHz}, \delta \mathrm{ppm} / \mathrm{CDCl}_{3}\right)$ : 7.29-7.18 (m, $4 \mathrm{H}), 5.22(\mathrm{~d}, J=12.0 \mathrm{~Hz}, 1 \mathrm{H}), 4.52(\mathrm{~d}, J=12.0 \mathrm{~Hz}, 1 \mathrm{H}), 3.18(\mathrm{~s}, 3 \mathrm{H})$, 2.70-2.56 (m, 1H), $2.51(\mathrm{~s}, 3 \mathrm{H}) 2.26-1.93(\mathrm{~m}, 3 \mathrm{H}), 1.86$ (dddd, $J=$ $18.7,8.8,4.4,1.6 \mathrm{~Hz}, 1 \mathrm{H}), 1.51-1.39(\mathrm{~m}, 1 \mathrm{H}) .{ }^{13} \mathrm{C}$ NMR $(75 \mathrm{MHz}, \delta$ $\left.\mathrm{ppm} / \mathrm{CDCl}_{3}\right): 216.2(\mathrm{C}), 171.0(\mathrm{C}), 133.5(\mathrm{C}), 129.3(\mathrm{CH}), 129.1(2 \mathrm{CH})$, $127.5(2 \mathrm{CH}), 70.7(\mathrm{CH}), 63.8(\mathrm{C}), 39.2\left(\mathrm{CH}_{2}\right), 32.0\left(\mathrm{CH}_{3}\right), 30.2\left(\mathrm{CH}_{2}\right)$, $19.8\left(\mathrm{CH}_{2}\right)$.

Pyrazolidinone $2 \mathrm{~h}$ and Acylhydrazone $\mathbf{6 h}$ : Following the general procedure with ethyl glyoxalate $(50 \%$ in toluene, $716 \mu \mathrm{L}$, $3.62 \mathrm{mmol})$, methylhydrazine $(213 \mu \mathrm{L}, 4.07 \mathrm{mmol})$ and 2-diazo-1,3cyclohexanedione ( $500 \mathrm{mg}, 3.62 \mathrm{mmol}$ ) in $9 \mathrm{~mL}$ of anhydrous toluene at $180{ }^{\circ} \mathrm{C}$ for $25 \mathrm{~min}$, and using AcOEt/PE, 4:6 as eluent for the flash chromatography, $\mathbf{6} \mathbf{h}$ and $\mathbf{2} \mathbf{h}$ were obtained, in that order, as white solids (6h: $490 \mathrm{mg}, 56 \%$; $\mathbf{2 h}: 87 \mathrm{mg}, 10 \%)$. For $\mathbf{6 h}: R_{\mathrm{f}}$ (EtOAc/ $\mathrm{PE}, 4: 6)=0.54 . \mathrm{Mp} 68.3-70.3{ }^{\circ} \mathrm{C}$. HRMS $\left.(\mathrm{ESI})^{+}\right):\left[\mathrm{M}+\mathrm{H}^{+}\right.$calcd. for $\mathrm{C}_{11} \mathrm{H}_{17} \mathrm{~N}_{2} \mathrm{O}_{4}{ }^{+} 241.1183$, found 241.1182. ${ }^{1} \mathrm{H}$ NMR $(400 \mathrm{MHz}$, $\delta \mathrm{ppm} /$ $\left.\mathrm{CDCl}_{3}\right): 7.00(\mathrm{~s}, 1 \mathrm{H}) 4.30(\mathrm{q}, J=7.1 \mathrm{~Hz}, 2 \mathrm{H}), 3.97(\mathrm{dd}, J=9.8,9.8 \mathrm{~Hz}$, $1 \mathrm{H}), 3.29(\mathrm{~s}, 3 \mathrm{H}), 2.70(\mathrm{ddd}, J=19.4,9.8,9.8 \mathrm{~Hz}, 1 \mathrm{H}), 2.35(\mathrm{~m}, 3 \mathrm{H})$, $2.21(\mathrm{~s}, 1 \mathrm{H}) 1.99-1.80(\mathrm{~m}, 1 \mathrm{H}), 1.34(\mathrm{t}, J=7.1 \mathrm{~Hz}, 3 \mathrm{H}) .{ }^{13} \mathrm{C}$ NMR $\left(75 \mathrm{MHz}, \delta \mathrm{ppm} / \mathrm{CDCl}_{3}\right): 213.3(\mathrm{C}), 172.3(\mathrm{C}), 162.7(\mathrm{C}), 129.2(\mathrm{CH})$, $61.4\left(\mathrm{CH}_{2}\right), 54.5(\mathrm{CH}), 37.7\left(\mathrm{CH}_{2}\right), 28.6\left(\mathrm{CH}_{3}\right), 27.9\left(\mathrm{CH}_{2}\right), 21.4\left(\mathrm{CH}_{2}\right)$, $14.2\left(\mathrm{CH}_{3}\right)$. For $2 \mathrm{~h}: R_{\mathrm{f}}(\mathrm{AcOEt} / \mathrm{PE}, 4: 6)=0.27 . \mathrm{Mp} 98.4-100.4^{\circ} \mathrm{C}$. HRMS $\left.(\mathrm{ESI})^{+}\right):[\mathrm{M}+\mathrm{H}]^{+}$calcd. for $\mathrm{C}_{11} \mathrm{H}_{17} \mathrm{~N}_{2} \mathrm{O}_{4}{ }^{+} 241.1183$, found 241.1183 . ${ }^{1} \mathrm{H}$ NMR $\left(300 \mathrm{MHz}, \delta \mathrm{ppm} / \mathrm{CDCl}_{3}\right): 5.13(\mathrm{~d}, J=11.3 \mathrm{~Hz}, 1 \mathrm{H}), 4.24-$ $4.10(\mathrm{~m}, 3 \mathrm{H}), 3.04(\mathrm{~s}, 3 \mathrm{H}), 2.62-2.48(\mathrm{~m}, 1 \mathrm{H}), 2.42-2.10(\mathrm{~m}, 4 \mathrm{H}), 2.15-$ $1.98(\mathrm{~m}, 1 \mathrm{H}) .{ }^{13} \mathrm{C}$ NMR $\left(75 \mathrm{MHz}, \delta \mathrm{ppm} / \mathrm{CDCl}_{3}\right): 215.5(\mathrm{C}), 168.8(\mathrm{C})$, $168.0(\mathrm{C}), 65.1(\mathrm{CH}), 62.1\left(\mathrm{CH}_{2}\right), 60.1(\mathrm{C}), 38.6\left(\mathrm{CH}_{2}\right), 31.8\left(\mathrm{CH}_{2}\right), 31.7$ $\left(\mathrm{CH}_{3}\right), 19.9\left(\mathrm{CH}_{2}\right), 14.1\left(\mathrm{CH}_{3}\right)$.

Pyrazolidinone 2i and Acylhydrazone 6i: Following the general procedure with benzyl glyoxalate $(188 \mathrm{mg}, 1.14 \mathrm{mmol})$, methylhydrazine $(66 \mu \mathrm{L}, 1.26 \mathrm{mmol}$ ) and 2-diazo-1,3-cyclohexanedione (220 mg, $1.14 \mathrm{mmol}$ ) in $3 \mathrm{~mL}$ of anhydrous toluene at $180{ }^{\circ} \mathrm{C}$ for $35 \mathrm{~min}$, and using AcOEt/PE, 4:6 as eluent for the flash chromatography, $\mathbf{6 i}$ and $\mathbf{2 i}$ were obtained, in that order, as white solids (6i: $226 \mathrm{mg}, 65 \%$; 2i:79 mg, 23 \%). For 6i: $R_{\mathrm{f}}(\mathrm{EtOAc} / \mathrm{PE}, 4: 6)=0.65$. Mp 107.5-108.5 ${ }^{\circ} \mathrm{C}$. HRMS $\left(\mathrm{ESI}^{+}\right)$: $\left[\mathrm{M}+\mathrm{H}^{+}\right.$calcd. for $\mathrm{C}_{16} \mathrm{H}_{19} \mathrm{~N}_{2} \mathrm{O}_{4}{ }^{+}$ 303.1339, found 303.1339. ${ }^{1} \mathrm{H}$ NMR $\left(300 \mathrm{MHz}, \delta \mathrm{ppm} / \mathrm{CDCl}_{3}\right): 7.41-$ $7.35(\mathrm{~m}, 5 \mathrm{H}), 7.03(\mathrm{~s}, 1 \mathrm{H}), 5.33-5.22(\mathrm{~m}, 2 \mathrm{H}), 3.95(\mathrm{dd}, J=10.0$, $10.0 \mathrm{~Hz}, 2 \mathrm{H}), 3.28(\mathrm{~s}, 3 \mathrm{H}), 2.63-2.49(\mathrm{~m}, 1 \mathrm{H}), 2.36-2.26(\mathrm{~m}, 2 \mathrm{H}), 2.26-$ $2.17(\mathrm{~m}, 1 \mathrm{H}), 2.16-2.04(\mathrm{~m}, 1 \mathrm{H}), 1.91-1.73(\mathrm{~m}, 1 \mathrm{H}) .{ }^{13} \mathrm{C}$ NMR $(75 \mathrm{MHz}$, $\left.\delta \mathrm{ppm} / \mathrm{CDCl}_{3}\right)$ : $213.1(\mathrm{C}), 172.3(\mathrm{C}), 162.4(\mathrm{C}), 135.2(\mathrm{C}), 128.7(\mathrm{CH})$, $128.6(2 \mathrm{CH}), 128.5(\mathrm{CH}), 128.4(2 \mathrm{CH}), 67.1\left(\mathrm{CH}_{2}\right), 54.5(\mathrm{CH}), 37.6\left(\mathrm{CH}_{2}\right)$, $28.6\left(\mathrm{CH}_{3}\right), 27.9\left(\mathrm{CH}_{2}\right), 21.3\left(\mathrm{CH}_{2}\right)$. The structure of $6 \mathbf{i}$ was further ascertained by COSY and HSQC $2 \mathrm{D}$ NMR experiments. For $2 \mathbf{i}: R_{\mathrm{f}}$ $\left(\right.$ AcOEt/PE, 4:6) $=0.25 . \mathrm{Mp} 147.0-149.0{ }^{\circ} \mathrm{C}$. HRMS $\left(E S I^{+}\right):[\mathrm{M}+\mathrm{H}]^{+}$ calcd. for $\mathrm{C}_{16} \mathrm{H}_{19} \mathrm{~N}_{2} \mathrm{O}_{4}{ }^{+} 303.1339$, found 303.1339. ${ }^{1} \mathrm{H} \mathrm{NMR}(300 \mathrm{MHz}$, $\left.\delta \mathrm{ppm} / \mathrm{CDCl}_{3}\right)$ : 7.31-7.37, (m, 5H), 5.25, (d, J=11.6 Hz, 1H), 5.18, (d, $J=11.0 \mathrm{~Hz}, 1 \mathrm{H}), 5.09(\mathrm{~d}, J=11.6 \mathrm{~Hz}, 1 \mathrm{H}), 4.19(\mathrm{~d}, J=11.0 \mathrm{~Hz}, 1 \mathrm{H})$, $3.06(\mathrm{~s}, 3 \mathrm{H}), 2.58-2.49(\mathrm{~m}, 1 \mathrm{H}), 2.32-2.06(\mathrm{~m}, 3 \mathrm{H}), 1.99-1.81(\mathrm{~m}, 2 \mathrm{H})$. ${ }^{13} \mathrm{C} \mathrm{NMR}\left(75 \mathrm{MHz}, \delta \mathrm{ppm} / \mathrm{CDCl}_{3}\right): 215.3$ (C), 168.8 (C), 167.9 (C), $134.5(\mathrm{C}), 129.0(\mathrm{CH}), 129.0(2 \mathrm{CH}), 128.8(2 \mathrm{CH}), 67.8\left(\mathrm{CH}_{2}\right), 65.1(\mathrm{CH})$ 
$60.6(\mathrm{C}), 38.4\left(\mathrm{CH}_{2}\right), 31.7\left(\mathrm{CH}_{3}\right), 31.7\left(\mathrm{CH}_{2}\right), 19.8\left(\mathrm{CH}_{2}\right)$. The structure of $\mathbf{2 i}$ was further ascertained by COSY, HSQC and HMBC 2D NMR experiments.

Pyrazolidinone 2j: Following the general procedure with phenylacetaldehyde $(423 \mu \mathrm{L}, 3.80 \mathrm{mmol})$, methylhydrazine $(213 \mu \mathrm{L}$, $4.07 \mathrm{mmol}$ ) and 2-diazo-1,3-cyclohexanedione (500 mg, $3.62 \mathrm{mmol}$ ) in $9 \mathrm{~mL}$ of anhydrous toluene at $180^{\circ} \mathrm{C}$ for $35 \mathrm{~min}$, and using EtOAc/ $\mathrm{PE}, 3: 7$ as eluent for the flash chromatography, $\mathbf{2} \mathbf{j}$ was obtained as a colorless oil $(700 \mathrm{mg}, 75 \%)$. The ${ }^{1} \mathrm{H}$ NMR analysis of the crude material showed that no more than $3 \%$ of acylhydrazone $\mathbf{6 j}$ (not isolated) was formed. $R_{\mathrm{f}}(\mathrm{AcOEt} / \mathrm{PE}, 3: 7)=0.14$. $\mathrm{HRMS}\left(\mathrm{ESI}^{+}\right)$: $[\mathrm{M}+$ $\mathrm{H}]^{+}$calcd. for $\mathrm{C}_{15} \mathrm{H}_{19} \mathrm{~N}_{2} \mathrm{O}_{2}{ }^{+}$259.1441, found 259.1440. ${ }^{1} \mathrm{H}$ NMR $\left(300 \mathrm{MHz}, \delta \mathrm{ppm} / \mathrm{CDCl}_{3}\right): 7.35-7.25(\mathrm{~m}, 3 \mathrm{H}), 7.21-7.16(\mathrm{~m}, 2 \mathrm{H}), 4.60$ $(\mathrm{d}, J=12.1 \mathrm{~Hz}, 1 \mathrm{H}), 3.79$ (ddd, $J=12.1,8.1,6.8 \mathrm{~Hz}, 1 \mathrm{H}), 3.00-2.83$ (m, 2H), 2.51-2.29 (m, 2H), 2.28-2.05 (m, 2H), 1.65-1.51 (m, 2H). ${ }^{13} \mathrm{C}$ NMR (75 MHz, $\left.\delta \mathrm{ppm} / \mathrm{CDCl}_{3}\right): 216.7(\mathrm{C}), 171.4$ (C), 136.7 (C), 129.0 $(2 \mathrm{CH}), 128.9(2 \mathrm{CH}), 127.3(\mathrm{CH}), 66.7(\mathrm{CH}), 61.3(\mathrm{C}), 40.0\left(\mathrm{CH}_{2}\right), 34.2$ $\left(\mathrm{CH}_{2}\right), 31.8\left(\mathrm{CH}_{3}\right), 30.9\left(\mathrm{CH}_{2}\right), 20.0\left(\mathrm{CH}_{2}\right)$.

Pyrazolidinone 2k: A solution of tert-butylhydrazine hydrochloride (541 mg, $4.34 \mathrm{mmol})$, potassium bicarbonate $(750 \mathrm{mg}, 5.43 \mathrm{mmol}$ ) and benzaldehyde ( $366 \mu \mathrm{L}, 3.62 \mathrm{mmol}$ ) in $9 \mathrm{~mL}$ anhydrous dichloromethane under an argon atmosphere in a sealed tube containing a Teflon coated magnetic stirring bar was irradiated at $140{ }^{\circ} \mathrm{C}$ for 10 minutes, cooled down to $55^{\circ} \mathrm{C}$ by airflow and concentrated in vacuo. The resulting material was dissolved/suspended in $9 \mathrm{~mL}$ toluene and filtered. The liquid phase was placed under argon atmosphere in a sealed tube containing a Teflon coated magnetic stirring bar, 2-diazo-cyclohexan-1,3-dione (500 mg, $3.62 \mathrm{mmol}$ ) was added and this mixture was irradiated at $160{ }^{\circ} \mathrm{C}$ for $15 \mathrm{~min}$. The resulting reaction mixture was cooled down to $55^{\circ} \mathrm{C}$ by airflow, concentrated and purify by flash chromatography using EtOAc/PE, 1:9 as eluent to afford $\mathbf{2 k}$ as a white solid (500 $\mathbf{m g}, 48 \%$ ). The ${ }^{1} \mathrm{H}$ NMR analysis of the crude material showed that no more than $3 \%$ of acylhydrazone $6 \mathbf{k}$ (not isolated) was formed. $R_{\mathrm{f}}(\mathrm{AcOEt} / \mathrm{PE}, 1: 9)=0.30$. Mp 110.3-112.3 ${ }^{\circ} \mathrm{C}$. HRMS (ESI+): $[\mathrm{M}+\mathrm{H}]^{+}$calcd. for $\mathrm{C}_{17} \mathrm{H}_{23} \mathrm{~N}_{2} \mathrm{O}_{2}{ }^{+}$ calcd. 287.1754, found 287.1754. ${ }^{1} \mathrm{H}$ NMR $\left(400 \mathrm{MHz}, \delta \mathrm{ppm} / \mathrm{CDCl}_{3}\right)$ : 7.38-7.33 (m, 3H), 7.29-7.27 (m, 2H), $5.12(\mathrm{~d}, J=11.0 \mathrm{~Hz}, 1 \mathrm{H}), 4.37$ $(\mathrm{d}, J=11.0 \mathrm{~Hz}, 1 \mathrm{H}), 2.66-2.53(\mathrm{~m}, 1 \mathrm{H}), 2.18-2.06(\mathrm{~m}, 1 \mathrm{H}), 2.04-1.87$, $(\mathrm{m}, 2 \mathrm{H}), 1.80-1.70,(\mathrm{~m}, 1 \mathrm{H}), 1.51,(\mathrm{~s}, 9 \mathrm{H}), 1.50-1.42(\mathrm{~m}, 1 \mathrm{H}) .{ }^{13} \mathrm{C}$ NMR (101 MHz, $\delta$ ppm/ $\left.\mathrm{CDCl}_{3}\right): 216.5(\mathrm{C}), 171.0(\mathrm{C}), 133.9(\mathrm{C}), 123.1(\mathrm{CH})$, $129.03(2 \mathrm{CH}), 127.5(2 \mathrm{CH}), 70.5(\mathrm{CH}), 65.4(\mathrm{C}), 58.0(\mathrm{C}), 39.5\left(\mathrm{CH}_{2}\right)$, $30.0\left(\mathrm{CH}_{2}\right), 28.0\left(3 \mathrm{CH}_{3}\right), 19.8\left(\mathrm{CH}_{2}\right)$.

General Procedure for Carbamates 7: To a solution of pyrazolidinone 2 (1 equiv.) in THF (ca. $0.1 \mathrm{M}$ ) was added slowly the appropriate chloroformate (1.2-2 equiv.) follow by a $2 \mathrm{M}$ solution of sodium carbonate in water (2-3 equiv.). The resulting solution was stirred at room temperature until full conversion (monitored by TLC). The solution was diluted with brine and extracted 3 times with dichloromethane. The organic layers were combined, dried with anhydrous sodium sulfate, concentrated in vacuo and purified by flash chromatography.

Carbamate $7 \mathbf{a}_{\mathrm{Me}}$ : Following the general procedure with $\mathbf{2 b}$ (457 mg, $1.87 \mathrm{mmol})$, methylchloroformate $(290 \mu \mathrm{L}, 3.74 \mathrm{mmol})$ and a $2 \mathrm{M}$ solution of $\mathrm{Na}_{2} \mathrm{CO}_{3}(3.6 \mathrm{~mL}, 7.2 \mathrm{mmol})$ in $19 \mathrm{~mL}$ of THF for $1 \mathrm{~h} \mathrm{45}$, and using EtOAc/PE, 2:8 as eluent for the flash chromatography, $7 \mathrm{a}_{\mathrm{Me}}$ was obtained as a yellow solid (499 mg, $88 \%$ ). $R_{\mathrm{f}}$ (AcOEt/ $\mathrm{PE}, 2: 8)=0.21$. Mp 140.9-141.5 ${ }^{\circ} \mathrm{C}$. HRMS $\left(E S I^{+}\right):[\mathrm{M}+\mathrm{H}]^{+}$calcd. for $\mathrm{C}_{16} \mathrm{H}_{19} \mathrm{~N}_{2} \mathrm{O}_{4}{ }^{+}$303.1339, found 303.1341. ${ }^{1} \mathrm{H}$ NMR (300 MHz, $\delta \mathrm{ppm} /$ $\left.\mathrm{CDCl}_{3}\right): 7.40-7.25(\mathrm{~m}, 3 \mathrm{H}), 7.12-7.02(\mathrm{~m}, 2 \mathrm{H}), 5.33(\mathrm{~s}, 1 \mathrm{H}), 3.83(\mathrm{~s}$, $3 \mathrm{H}), 3.46(\mathrm{~s}, 3 \mathrm{H}), 2.52-2.39(\mathrm{~m}, 1 \mathrm{H}), 2.34-2.00(\mathrm{~m}, 5 \mathrm{H}) .{ }^{13} \mathrm{C}$ NMR $\left(75 \mathrm{MHz}, \delta \mathrm{ppm} / \mathrm{CDCl}_{3}\right): 210.6$ (C), 170.1 (C), 158.2 (C), 136.0 (C),
$128.9(2 \mathrm{CH}), 128.6(\mathrm{CH}), 126.2(2 \mathrm{CH}), 71.0(\mathrm{CH}), 63.2(\mathrm{C}), 54.1\left(\mathrm{CH}_{3}\right)$, $38.5\left(\mathrm{CH}_{2}\right), 35.2\left(\mathrm{CH}_{2}\right), 34.3\left(\mathrm{CH}_{3}\right), 20.0\left(\mathrm{CH}_{2}\right)$.

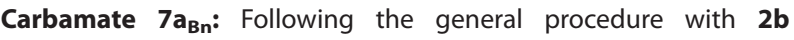
(1405 mg, $5.75 \mathrm{mmol})$, benzylchloroformate $(1.60 \mathrm{~mL}, 11.5 \mathrm{mmol})$ and a $2 \mathrm{M}$ solution of $\mathrm{Na}_{2} \mathrm{CO}_{3}(8.6 \mathrm{~mL}, 17.2 \mathrm{mmol})$ in $60 \mathrm{~mL}$ of THF for $4 \mathrm{~h}$, and using EtOAc/PE, 2:8 as eluent for the flash chromatography, $7 \mathrm{a}_{\mathrm{Bn}}$ was obtained as a white solid (1695 mg, $78 \%$ ). $R_{\mathrm{f}}$ (AcOEt/ $\mathrm{PE}, 2: 8)=0.15 . \mathrm{Mp} 101.1-102.1^{\circ} \mathrm{C}$. HRMS $\left.(\mathrm{ESI})^{+}\right):[\mathrm{M}+\mathrm{H}]^{+}$calcd. for $\mathrm{C}_{22} \mathrm{H}_{23} \mathrm{~N}_{2} \mathrm{O}_{4}{ }^{+} 379.1652$, found 379.1654. ' $\mathrm{H}$ NMR (300 MHz, $\delta \mathrm{ppm} /$ $\left.\mathrm{CDCl}_{3}\right)$ : 7.41-7.28 (m, 8H), $7.07(\mathrm{dd}, J=7.6,2.0 \mathrm{~Hz}, 2 \mathrm{H}), 5.32(\mathrm{~s}, 1 \mathrm{H})$, $5.28(\mathrm{~d}, J=12.2 \mathrm{~Hz}, 1 \mathrm{H}), 5.17(\mathrm{~d}, J=12.2 \mathrm{~Hz}, 1 \mathrm{H}), 3.45(\mathrm{~s}, 3 \mathrm{H}), 2.53-$ $2.29(\mathrm{~m}, 1 \mathrm{H}), 2.27-1.93(\mathrm{~m}, 5 \mathrm{H}) .{ }^{13} \mathrm{C}$ NMR $\left(75 \mathrm{MHz}, \delta \mathrm{ppm} / \mathrm{CDCl}_{3}\right)$ : 210.8 (C), 170.1 (C), 157.7 (C), 136.0 (C), 135.2 (C), 128.9 (2CH), 128.8 $(2 \mathrm{CH}), 128.8(\mathrm{CH}), 128.7(\mathrm{CH}), 128.3(2 \mathrm{CH}), 126.4(2 \mathrm{CH}), 71.0(\mathrm{CH})$, $68.9\left(\mathrm{CH}_{2}\right), 63.2(\mathrm{C}), 38.5\left(\mathrm{CH}_{2}\right), 34.9\left(\mathrm{CH}_{2}\right), 34.5\left(\mathrm{CH}_{3}\right), 19.9\left(\mathrm{CH}_{2}\right)$.

Carbamate $\mathbf{7} \mathrm{d}_{\mathrm{Me}}$ : Following the general procedure with $\mathbf{2 d}$ (527 mg, $1.82 \mathrm{mmol})$, methylchloroformate $(170 \mu \mathrm{L}, 2.19 \mathrm{mmol})$ and a $2 \mathrm{M}$ solution of $\mathrm{Na}_{2} \mathrm{CO}_{3}(1.8 \mathrm{~mL}, 3.6 \mathrm{mmol})$ in $19 \mathrm{~mL}$ of THF for $4 \mathrm{~h}$, and using EtOAc/ $\mathrm{CH}_{2} \mathrm{Cl}_{2}, 4: 6$ as eluent for the flash chromatography, $7 \mathrm{~d}_{\mathrm{Me}}$ was obtained as a yellow solid (632 mg, $\left.99 \%\right) . R_{\mathrm{f}}$ $\left(\right.$ AcOEt/DCM, 4:6) $=0.63 . \mathrm{Mp} 186.8-188.8^{\circ} \mathrm{C}$. HRMS $\left.(\mathrm{ESI})^{+}\right):[\mathrm{M}+\mathrm{H}]^{+}$ calcd. for $\mathrm{C}_{16} \mathrm{H}_{18} \mathrm{~N}_{3} \mathrm{O}_{6}{ }^{+} 348.1190$, found 348.1190 . ' $\mathrm{H}$ NMR (300 MHz, $\left.\delta \mathrm{ppm} / \mathrm{CDCl}_{3}\right): 8.20$ (d, J = $\left.9.0 \mathrm{~Hz}, 2 \mathrm{H}\right), 7.25(\mathrm{~d}, J=9.0 \mathrm{~Hz}, 2 \mathrm{H}), 5.39$ $(\mathrm{s}, 1 \mathrm{H}), 3.85(\mathrm{~s}, 3 \mathrm{H}), 3.47(\mathrm{~s}, 3 \mathrm{H}), 2.59-2.47(\mathrm{~m}, 1 \mathrm{H}), 2.39-2.20(\mathrm{~m}$, $3 \mathrm{H}), 2.18-2.01(\mathrm{~m}, 2 \mathrm{H}) .{ }^{13} \mathrm{C} \mathrm{NMR}\left(75 \mathrm{MHz}, \delta \mathrm{ppm} / \mathrm{CDCl}_{3}\right): 211.0(\mathrm{C})$, $169.4(\mathrm{C}), 158.0(\mathrm{C}), 147.8(\mathrm{C}), 143.2(\mathrm{C}), 127.4(2 \mathrm{CH}), 124.0(2 \mathrm{CH})$, $70.0(\mathrm{CH}), 62.9(\mathrm{C}), 54.4\left(\mathrm{CH}_{3}\right), 38.9\left(\mathrm{CH}_{2}\right), 35.4\left(\mathrm{CH}_{2}\right), 34.3\left(\mathrm{CH}_{3}\right), 20.1$ $\left(\mathrm{CH}_{2}\right)$.

Carbamate $\mathbf{7 f _ { \mathrm { Bn } }}$ : Following the general procedure with $\mathbf{2 f}(550 \mathrm{mg}$, $2.00 \mathrm{mmol})$, benzylchloroformate $(440 \mu \mathrm{L}, 3.10 \mathrm{mmol})$ and a $2 \mathrm{M}$ solution of $\mathrm{Na}_{2} \mathrm{CO}_{3}(2.5 \mathrm{~mL}, 5.0 \mathrm{mmol})$ in $20 \mathrm{~mL}$ of THF for $4 \mathrm{~h}$, and using EtOAc/PE, 2:8 as eluent for the flash chromatography, $\mathbf{7 f}_{\mathrm{Bn}}$ was obtained as a colorless oil (674 mg, $82 \%)$. $R_{\mathrm{f}}(\mathrm{AcOEt} / \mathrm{PE}, 2: 8)=$ 0.23. HRMS (ESI $\left.{ }^{+}\right):[\mathrm{M}+\mathrm{H}]^{+}$calcd. for $\mathrm{C}_{23} \mathrm{H}_{25} \mathrm{~N}_{2} \mathrm{O}_{5}{ }^{+} 409.1758$, found 409.1764. ${ }^{1} \mathrm{H}$ NMR $\left(300 \mathrm{MHz}, \delta \mathrm{ppm} / \mathrm{CDCl}_{3}\right): 7.38-7.33(\mathrm{~m}, 3 \mathrm{H}), 7.31-$ $7.25(\mathrm{~m}, 2 \mathrm{H}), 7.01(\mathrm{~d}, J=8.6 \mathrm{~Hz}, 2 \mathrm{H}), 6.84(\mathrm{~d}, J=8.6 \mathrm{~Hz}, 2 \mathrm{H}), 5.27$ $(\mathrm{d}, J=11.6 \mathrm{~Hz}, 1 \mathrm{H}), 5.25(\mathrm{~s}, 1 \mathrm{H}), 5.16(\mathrm{~d}, J=11.6 \mathrm{~Hz}, 1 \mathrm{H}), 3.78(\mathrm{~s}$, $3 \mathrm{H}), 3.43(\mathrm{~s}, 3 \mathrm{H}), 2.38(\mathrm{~m}, 1 \mathrm{H}), 2.12(\mathrm{~m}, 4 \mathrm{H}), 1.97(\mathrm{~m}, 1 \mathrm{H}) .{ }^{13} \mathrm{C}$ NMR $\left(75 \mathrm{MHz}, \delta \mathrm{ppm} / \mathrm{CDCl}_{3}\right): 210.8$ (C), 170.3 (C), 159.8 (C), 157.7 (C), $135.3(\mathrm{C}), 128.8(2 \mathrm{CH}), 128.8(\mathrm{CH}), 128.2(2 \mathrm{CH}), 128.2(\mathrm{C}), 127.8(2 \mathrm{CH})$, $114.3(2 \mathrm{CH}), 70.7(\mathrm{CH}), 68.9\left(\mathrm{CH}_{2}\right), 63.1(\mathrm{C}), 55.4\left(\mathrm{CH}_{3}\right), 38.4\left(\mathrm{CH}_{2}\right)$, $34.9\left(\mathrm{CH}_{2}\right), 34.6\left(\mathrm{CH}_{3}\right), 19.9\left(\mathrm{CH}_{2}\right)$.

Carbamate $\mathbf{7} \mathbf{g}_{\mathrm{Me}}$ : Following the general procedure with $\mathbf{2 g}$ $(502 \mathrm{mg}, 1.94 \mathrm{mmol})$, methylchloroformate $(180 \mu \mathrm{L}, 2.32 \mathrm{mmol})$ and a $2 \mathrm{M}$ solution of $\mathrm{Na}_{2} \mathrm{CO}_{3}(1.9 \mathrm{~mL}, 3.8 \mathrm{mmol})$ in $19 \mathrm{~mL}$ of THF for $2 \mathrm{~h}$, and using EtOAc/PE, 4:6 as eluent for the flash chromatography, $7 \mathbf{g}_{\text {Me }}$ was obtained as a white solid $(607 \mathrm{mg}, 99 \%) . R_{\mathrm{f}}$ (AcOEt/PE, 4:6) $=0.25$. Mp 157.3-159.0 ${ }^{\circ} \mathrm{C}$. HRMS $\left(\mathrm{ESI}^{+}\right):\left[\mathrm{M}+\mathrm{H}^{+}\right.$calcd. for $\mathrm{C}_{17} \mathrm{H}_{21} \mathrm{~N}_{2} \mathrm{O}_{4}{ }^{+}$317.1496, found 317.1496. ' $\mathrm{H}$ NMR $(300 \mathrm{MHz}, \delta \mathrm{ppm} /$ $\mathrm{CDCl}_{3}$ ): 7.25-7.17 (m, 2H), 7.10 (ddd, J = 6.5, 6.5, $\left.4.0 \mathrm{~Hz}, 2 \mathrm{H}\right), 5.58$ $(\mathrm{s}, 1 \mathrm{H}), 3.84(\mathrm{~s}, 3 \mathrm{H}), 3.44(\mathrm{~s}, 3 \mathrm{H}), 2.57-2.44(\mathrm{~m}, 1 \mathrm{H}), 2.39-2.16(\mathrm{~m}, 4 \mathrm{H})$, 2.14-2.01 (m, 1H). ${ }^{13} \mathrm{C}$ NMR $\left(75 \mathrm{MHz}, \delta \mathrm{ppm} / \mathrm{CDCl}_{3}\right): 209.9$ (C), 170.3 (C), $158.1(\mathrm{C}), 134.1(\mathrm{C}), 133.9(\mathrm{C}), 130.5(\mathrm{CH}), 128.4(\mathrm{CH}), 126.5(\mathrm{CH})$, 126.1 (CH), $68.1(\mathrm{CH}), 61.8(\mathrm{C}), 54.1\left(\mathrm{CH}_{3}\right), 38.9\left(\mathrm{CH}_{2}\right), 35.8\left(\mathrm{CH}_{2}\right), 33.8$ $\left(\mathrm{CH}_{3}\right), 20.1\left(\mathrm{CH}_{2}\right), 19.5\left(\mathrm{CH}_{3}\right)$.

Carbamate $7 \mathbf{h}_{\mathbf{B n}}$ : Following the general procedure with $\mathbf{2 h}$ $(690 \mathrm{mg}, 2.87 \mathrm{mmol})$, benzylchloroformate $(490 \mu \mathrm{L}, 3.45 \mathrm{mmol})$ and a $2 \mathrm{M}$ solution of $\mathrm{Na}_{2} \mathrm{CO}_{3}(2.9 \mathrm{~mL}, 5.8 \mathrm{mmol})$ in $28 \mathrm{~mL}$ of THF for $4 \mathrm{~h}$, and using EtOAc/PE, 4:6 as eluent for the flash chromatography, $7 \mathbf{h}_{\mathrm{Bn}}$ was obtained as a colorless oil $(858 \mathrm{mg}, 80 \%) . R_{\mathrm{f}}$ (AcOEt/PE, 4:6) $=0.64$. HRMS $\left(E S I^{+}\right):[M+H]^{+}$calcd. for $\mathrm{C}_{19} \mathrm{H}_{23} \mathrm{~N}_{2} \mathrm{O}_{6}{ }^{+} 375.1551$, 
found 375.1549. ${ }^{1} \mathrm{H}$ NMR $\left(300 \mathrm{MHz}, \delta \mathrm{ppm} / \mathrm{CDCl}_{3}\right): 7.45-7.32(\mathrm{~m}$, $5 \mathrm{H}), 5.30(\mathrm{~d}, J=12.1 \mathrm{~Hz}, 1 \mathrm{H}), 5.20(\mathrm{~d}, J=12.1 \mathrm{~Hz}, 1 \mathrm{H}), 4.81(\mathrm{~s}, 1 \mathrm{H})$, $4.18(\mathrm{dq}, J=11.8,7.1 \mathrm{~Hz}, 2 \mathrm{H}), 3.30(\mathrm{~s}, 3 \mathrm{H}), 2.51-2.27(\mathrm{~m}, 3 \mathrm{H}), 2.23-$ $2.13(\mathrm{~m}, 1 \mathrm{H}), 2.10-1.93(\mathrm{~m}, 2 \mathrm{H}), 1.26(\mathrm{t}, J=7.1 \mathrm{~Hz}, 3 \mathrm{H}) .{ }^{13} \mathrm{C}$ NMR $\left(101 \mathrm{MHz}, \delta \mathrm{ppm} / \mathrm{CDCl}_{3}\right): 211.1$ (C), 170.5 (C), 167.1 (C), 156.7 (C), $135.0(\mathrm{C}), 128.9(\mathrm{CH}), 128.9(2 \mathrm{CH}), 128.4(2 \mathrm{CH}), 69.2\left(\mathrm{CH}_{2}\right), 67.9(\mathrm{CH})$ $62.2\left(\mathrm{CH}_{2}\right), 60.9(\mathrm{C}), 38.7\left(\mathrm{CH}_{2}\right), 34.7\left(\mathrm{CH}_{3}\right), 34.7\left(\mathrm{CH}_{2}\right), 20.0\left(\mathrm{CH}_{2}\right), 14.0$ $\left(\mathrm{CH}_{3}\right)$. The structure of $\mathbf{7} \mathbf{h}_{\mathbf{B n}}$ was further ascertained by an HSQC 2D NMR experiment.

Carbamate $7 \mathbf{i}_{\mathrm{Bn}}$ : Following the general procedure with 2i (1580 mg, $5.23 \mathrm{mmol})$, benzylchloroformate $(890 \mu \mathrm{L}, 6.27 \mathrm{mmol})$ and a $2 \mathrm{M}$ solution of $\mathrm{Na}_{2} \mathrm{CO}_{3}(6.5 \mathrm{~mL}, 13.0 \mathrm{mmol})$ in $130 \mathrm{~mL}$ of THF for $4 \mathrm{~h}$, and using EtOAc/PE, 2:8 as eluent for the flash chromatography, $\mathbf{7} \mathbf{i}_{\mathbf{B n}}$ was obtained as a colorless oil $(1180 \mathrm{mg}, 52 \%)$. $R_{\mathrm{f}}(\mathrm{AcOEt} / \mathrm{PE}, 2: 8)=0.30$. HRMS $\left(\mathrm{ESI} \mathrm{I}^{+}\right):[\mathrm{M}+\mathrm{H}]^{+}$calcd. for $\mathrm{C}_{24} \mathrm{H}_{25} \mathrm{~N}_{2} \mathrm{O}_{6}{ }^{+}$ 437.1707, found 437.1709; $\left[\mathrm{M}+\mathrm{NH}_{4}\right]^{+}$calcd. for $\mathrm{C}_{24} \mathrm{H}_{28} \mathrm{~N}_{3} \mathrm{O}_{6}{ }^{+}$ 454.1973, found 454.1978. ${ }^{1} \mathrm{H}$ NMR $\left(300 \mathrm{MHz}, \delta \mathrm{ppm} / \mathrm{CDCl}_{3}\right): 7.43-$ $7.31(\mathrm{~m}, 10 \mathrm{H}), 5.29(\mathrm{~d}, J=11.9 \mathrm{~Hz}, 1 \mathrm{H}), 5.18(\mathrm{~d}, J=12.3 \mathrm{~Hz}, 2 \mathrm{H})$ $5.06(\mathrm{~d}, J=11.9 \mathrm{~Hz}, 1 \mathrm{H}), 4.85(\mathrm{~s}, 1 \mathrm{H}), 3.24(\mathrm{~s}, 3 \mathrm{H}), 2.50-2.24(\mathrm{~m}, 3 \mathrm{H})$ $2.23-1.88(\mathrm{~m}, 3 \mathrm{H}) .{ }^{13} \mathrm{C}$ NMR $\left(75 \mathrm{MHz}, \delta \mathrm{ppm} / \mathrm{CDCl}_{3}\right): 211.2(\mathrm{C}), 170.4$ (C), 167.0 (C), 156.9 (C), 135.0 (C), $134.8(\mathrm{C}), 129.0(\mathrm{CH}), 129.0(2 \mathrm{CH})$, $128.9(2 \mathrm{CH}), 128.8(\mathrm{CH}), 128.8(2 \mathrm{CH}), 128.5(2 \mathrm{CH}), 69.3\left(\mathrm{CH}_{2}\right), 68.2$ $\left(\mathrm{CH}_{2}\right), 68.0(\mathrm{CH}), 61.0(\mathrm{C}), 38.7\left(\mathrm{CH}_{2}\right), 34.8\left(\mathrm{CH}_{2}\right), 34.7\left(\mathrm{CH}_{3}\right), 20.0$ $\left(\mathrm{CH}_{2}\right)$.

Carbamate $7 \mathbf{j}_{\mathrm{B}_{\mathrm{n}}}$ : Following the general procedure with $\mathbf{2 j} \mathbf{j} 600 \mathrm{mg}$ $2.32 \mathrm{mmol})$, benzylchloroformate $(660 \mu \mathrm{L}, 4.65 \mathrm{mmol})$ and a $2 \mathrm{M}$ solution of $\mathrm{Na}_{2} \mathrm{CO}_{3}(2.9 \mathrm{~mL}, 5.8 \mathrm{mmol})$ in $20 \mathrm{~mL}$ of THF for $4 \mathrm{~h}$, and using EtOAc/PE, 2:8 as eluent for the flash chromatography, $\mathbf{7 j}_{\mathbf{B n}_{n}}$ was obtained as a yellowish solid $(711 \mathrm{mg}, 78 \%) . R_{\mathrm{f}}$ (ACOEt/PE, 2:8) $=0.28$. HRMS (ESI+): $[\mathrm{M}+\mathrm{H}]^{+}$calcd. for $\mathrm{C}_{23} \mathrm{H}_{25} \mathrm{~N}_{2} \mathrm{O}_{4}{ }^{+} 393.1809$, found 393.1809. ${ }^{1} \mathrm{H}$ NMR $\left(300 \mathrm{MHz}, \delta \mathrm{ppm} / \mathrm{CDCl}_{3}\right): 7.43-7.09(\mathrm{~m}$, $10 \mathrm{H}), 5.17-5.08(\mathrm{~m}, 2 \mathrm{H}), 4.60(\mathrm{dd}, \mathrm{J}=7.0,7.0 \mathrm{~Hz}, 1 \mathrm{H}), 3.27(\mathrm{~s}, 3 \mathrm{H})$, 3.07-2.87 $(\mathrm{m}, 2 \mathrm{H}), 2.42-1.88(\mathrm{~m}, 5 \mathrm{H}), 1.83-1.68(\mathrm{~m}, 1 \mathrm{H}) .{ }^{13} \mathrm{C}$ NMR (75 MHz, $\left.\delta \mathrm{ppm} / \mathrm{CDCl}_{3}\right): 212.7$ (C), 170.9 (C), 157.95 (C), 136.79 (C), $135.26(\mathrm{C}), 129.8(2 \mathrm{CH}), 128.8(2 \mathrm{CH}), 128.7(\mathrm{CH}), 128.5(2 \mathrm{CH}), 128.2$ $(2 \mathrm{CH}), 126.9(\mathrm{CH}), 69.0(\mathrm{CH}), 68.7\left(\mathrm{CH}_{2}\right), 61.3(\mathrm{C}), 39.3\left(\mathrm{CH}_{2}\right), 36.7$ $\left(\mathrm{CH}_{2}\right), 34.5\left(\mathrm{CH}_{2}\right), 34.1\left(\mathrm{CH}_{3}\right), 20.0\left(\mathrm{CH}_{2}\right)$.

General Procedure for Triflyl Enol Ethers 8: To a solution of ketone 7 ( 1 equiv.) and $\mathrm{Tf}_{2} \mathrm{NPh}$ (1.2 equiv.) in anhydrous THF (ca. $0.1 \mathrm{M}$ ) at $-80{ }^{\circ} \mathrm{C}$ was added a $0.5 \mathrm{M}$ solution of KHMDS in toluene (1.5 equiv.). The solution was stirred at this temperature during $1 \mathrm{~h}$ then warmed to room temperature and stirred for an additional $2 \mathrm{~h}$ at this temperature. The resulting mixture was poured into a saturated solution of ammonium chloride and extracted with three times with dichloromethane. The organic layers were combined, dried with anhydrous sodium sulfate and concentrated in vacuo to give the crude product, which was purified by flash chromatography.

Triflyl Enol Ether $\mathbf{8} \mathbf{a}_{\mathrm{Me}}$ : Following the general procedure with $\mathbf{7} \mathrm{a}_{\mathrm{Me}}$ (456 mg, $1.51 \mathrm{mmol}$ ), $\mathrm{Tf}_{2} \mathrm{NPh}(653 \mathrm{mg}, 1.83 \mathrm{mmol}$ ) and KHMDS $0.5 \mathrm{M}$ in toluene ( $4.8 \mathrm{~mL}, 2.4 \mathrm{mmol})$ in $17 \mathrm{~mL}$ of anhydrous THF, and using $\mathrm{EtOAc/PE}, 4: 6$ as eluent for the flash chromatography, $\mathbf{8} \mathbf{a}_{\mathrm{me}}$ was obtained as a white solid (583 mg, $89 \%) . R_{\mathrm{f}}(\mathrm{EtOAc} / \mathrm{PE}, 4: 6)=0.50$. Mp 181.2-181.7 ${ }^{\circ} \mathrm{C}$. HRMS (ESI+): $[\mathrm{M}+\mathrm{H}]^{+}$calcd. for $\mathrm{C}_{17} \mathrm{H}_{18} \mathrm{~F}_{3} \mathrm{~N}_{2} \mathrm{O}_{6} \mathrm{~S}^{+}$ 435.0832, found 435.0832. ${ }^{~} \mathrm{H}$ NMR $\left(300 \mathrm{MHz}, \delta \mathrm{ppm} / \mathrm{CDCl}_{3}\right): 7.40$ $7.31(\mathrm{~m}, 3 \mathrm{H}), 7.09(\mathrm{dd}, J=7.2,2.5 \mathrm{~Hz}, 2 \mathrm{H}), 5.88(\mathrm{~s}, 1 \mathrm{H}), 5.38(\mathrm{~s}, 1 \mathrm{H})$, $3.85(\mathrm{~s}, 3 \mathrm{H}), 3.48(\mathrm{~s}, 3 \mathrm{H}), 2.74-2.39(\mathrm{~m}, 3 \mathrm{H}), 2.19(\mathrm{dd}, J=11.8,7.1 \mathrm{~Hz}$, 1H). ${ }^{13} \mathrm{C}$ NMR (75 MHz, $\left.\delta \mathrm{ppm} / \mathrm{CDCl}_{3}\right): 169.8$ (C), 158.2 (C), 144.9 (C), $135.6(\mathrm{C}), 129.0(2 \mathrm{CH}), 128.8(\mathrm{CH}), 126.0(2 \mathrm{CH}), 120.0(\mathrm{CH}), 118.1(\mathrm{q}$ $\left.J=323 \mathrm{~Hz}, \mathrm{CF}_{3}\right), 70.0(\mathrm{CH}), 61.8(\mathrm{C}), 54.2\left(\mathrm{CH}_{3}\right), 35.0\left(\mathrm{CH}_{2}\right), 33.9\left(\mathrm{CH}_{3}\right)$, $26.4\left(\mathrm{CH}_{2}\right) .{ }^{19} \mathrm{~F}$ NMR $\left(282 \mathrm{MHz}, \delta \mathrm{ppm} / \mathrm{CDCl}_{3}\right):-74.1(\mathrm{~s}, 3 \mathrm{~F})$.
Triflyl Enol Ether $\mathbf{8 a}_{\mathbf{B} \mathbf{n}}$ : Following the general procedure with $7 \mathbf{a}_{\mathbf{B n}}$ (800 mg, $2.11 \mathrm{mmol}), \mathrm{Tf}_{2} \mathrm{NPh}(863 \mathrm{mg}, 2.42 \mathrm{mmol})$ and KHMDS $0.5 \mathrm{M}$ in toluene (6.2 mL, $3.1 \mathrm{mmol}$ ) in $21 \mathrm{~mL}$ of anhydrous THF, and using EtOAc/PE, 2:8 as eluent for the flash chromatography, $\mathbf{8} \mathbf{a}_{\text {me }}$ was obtained as a white solid (908 mg, $84 \%) . R_{\mathrm{f}}(\mathrm{AcOEt} / \mathrm{PE}, 2: 8)=0.52$. Mp 134.5-136.5 ${ }^{\circ} \mathrm{C}$. HRMS (ESI+): $[\mathrm{M}+\mathrm{H}]^{+}$calcd. for $\mathrm{C}_{23} \mathrm{H}_{22} \mathrm{~F}_{3} \mathrm{~N}_{2} \mathrm{O}_{6} \mathrm{~S}^{+}$ 511.1145, found 511.1146. ${ }^{1} \mathrm{H}$ NMR $\left(400 \mathrm{MHz}, \delta \mathrm{ppm} / \mathrm{CDCl}_{3}\right): 7.46-$ $7.30(\mathrm{~m}, 8 \mathrm{H}), 7.12-7.06(\mathrm{~m}, 2 \mathrm{H}), 5.85(\mathrm{dd}, J=2.6,2.6 \mathrm{~Hz}, 1 \mathrm{H}), 5.37$ $(\mathrm{s}, 1 \mathrm{H}), 5.32(\mathrm{~d}, J=12.1 \mathrm{~Hz}, 1 \mathrm{H}), 5.19(\mathrm{~d}, J=12.2 \mathrm{~Hz}, 1 \mathrm{H}), 3.46(\mathrm{~s}$, $3 \mathrm{H}), 2.64-2.32(\mathrm{~m}, 3 \mathrm{H}), 2.10-1.96(\mathrm{~m}, 1 \mathrm{H}) .{ }^{13} \mathrm{C}$ NMR $(101 \mathrm{MHz}, \delta$ $\mathrm{ppm} / \mathrm{CDCl} 3$ ): 169.8 (C), 157.7 (C), 144.8 (C), 135.6 (C), 135.2 (C), 128.9 $(2 \mathrm{CH}), 128.9(\mathrm{CH}), 128.8(2 \mathrm{CH}), 128.7(\mathrm{CH}), 128.3(2 \mathrm{CH}), 126.0(2 \mathrm{CH})$, $119.9(\mathrm{CH}), 118.1\left(\mathrm{q}, J=321 \mathrm{~Hz}, \mathrm{CF}_{3}\right), 70.13(\mathrm{CH}), 69.0\left(\mathrm{CH}_{2}\right), 61.8$ (C), $34.7\left(\mathrm{CH}_{2}\right), 34.0\left(\mathrm{CH}_{3}\right), 26.2\left(\mathrm{CH}_{2}\right) .{ }^{19} \mathrm{~F}$ NMR $(282 \mathrm{MHz}, \delta \mathrm{ppm} /$ $\left.\mathrm{CDCl}_{3}\right):-74.08(\mathrm{~s}, 3 \mathrm{~F})$.

Triflyl Enol Ether $\mathbf{8} \mathbf{d}_{\mathrm{Me}}$ : Following the general procedure with $\mathbf{7} \mathbf{d}_{\mathrm{Me}}$ (541 mg, $1.56 \mathrm{mmol}$ ), Tf 2 NPh (568 mg, $1.59 \mathrm{mmol}$ ) and KHMDS $0.5 \mathrm{M}$ in toluene ( $4.4 \mathrm{~mL}, 2.2 \mathrm{mmol}$ ) in $16 \mathrm{~mL}$ of anhydrous THF, and using EtOAc/ $\mathrm{CH}_{2} \mathrm{Cl}_{2}, 1: 9$ as eluent for the flash chromatography, $\mathbf{8} \mathbf{d}_{\mathbf{M e}}$ was obtained as a white solid (528 mg, $71 \%) . R_{\mathrm{f}}\left(\mathrm{EtOAc} / \mathrm{CH}_{2} \mathrm{Cl}_{2}, 3: 7\right)=$ 0.83. Mp 152.0-154.0 ${ }^{\circ} \mathrm{C}$. HRMS (ESI+): $[\mathrm{M}+\mathrm{H}]^{+}$calcd. for $\mathrm{C}_{17} \mathrm{H}_{17} \mathrm{~F}_{3} \mathrm{~N}_{3} \mathrm{O}_{8} \mathrm{~S}^{+}$480.0683, found 480.0682. ${ }^{1} \mathrm{H}$ NMR $(400 \mathrm{MHz}$, $\delta$ $\left.\mathrm{ppm} / \mathrm{CDCl}_{3}\right): 8.24(\mathrm{~d}, J=8.7 \mathrm{~Hz}, 2 \mathrm{H}), 7.29(\mathrm{~d}, J=8.6 \mathrm{~Hz}, 3 \mathrm{H}), 5.93(\mathrm{~s}$, $1 \mathrm{H}), 5.48(\mathrm{~s}, 1 \mathrm{H}), 3.88(\mathrm{~s}, 3 \mathrm{H}), 3.50(\mathrm{~s}, 3 \mathrm{H}), 2.74-2.47(\mathrm{~m}, 3 \mathrm{H}), 2.27-$ $2.20(\mathrm{~m}, 1 \mathrm{H}) .{ }^{13} \mathrm{C}$ NMR $\left(101 \mathrm{MHz}, \delta \mathrm{ppm} / \mathrm{CDCl}_{3}\right): 169.0(\mathrm{C}), 158.1(\mathrm{C})$, $148.2(\mathrm{C}), 144.2(\mathrm{C}), 142.8(\mathrm{C}), 127.2(2 \mathrm{CH}), 124.4(2 \mathrm{CH}), 120.8(\mathrm{CH})$, $117.9\left(\mathrm{q}, J=322 \mathrm{~Hz}, \mathrm{CF}_{3}\right), 69.3(\mathrm{CH}), 61.8(\mathrm{C}), 54.6\left(\mathrm{CH}_{3}\right), 34.9\left(\mathrm{CH}_{2}\right)$, $34.1\left(\mathrm{CH}_{3}\right), 26.5\left(\mathrm{CH}_{2}\right) .{ }^{19} \mathrm{~F}$ NMR $\left(282 \mathrm{MHz}, \delta \mathrm{ppm} / \mathrm{CDCl}_{3}\right)$ : $-74.2(\mathrm{~s}$, 3F).

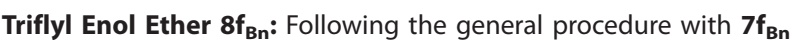
$(600 \mathrm{mg}, 1.47 \mathrm{mmol}), \mathrm{Tf}_{2} \mathrm{NPh}(672 \mathrm{mg}, 1.88 \mathrm{mmol})$ and KHMDS $0.5 \mathrm{M}$ in toluene $(4.4 \mathrm{~mL}, 2.2 \mathrm{mmol}$ ) in $15 \mathrm{~mL}$ of anhydrous THF, and using EtOAc/PE, 2:8 as eluent for the flash chromatography, $\mathbf{8 f}_{\mathrm{Bn}}$ was obtained as a white solid (580 mg, $73 \%) . R_{\mathrm{f}}(\mathrm{AcOEt} / \mathrm{PE}, 2: 8)=0.18$. Mp 103.9-105.9 ${ }^{\circ} \mathrm{C}$. HRMS (ESI+): $[\mathrm{M}+\mathrm{H}]^{+}$calcd. for $\mathrm{C}_{24} \mathrm{H}_{24} \mathrm{~F}_{3} \mathrm{~N}_{2} \mathrm{O}_{7} \mathrm{~S}^{+}$ 541.1251, found 541.1251. ${ }^{1} \mathrm{H} \mathrm{NMR}\left(300 \mathrm{MHz}, \delta \mathrm{ppm} / \mathrm{CDCl}_{3}\right): 7.40-$ $7.30(\mathrm{~m}, 5 \mathrm{H}), 7.01(\mathrm{~d}, J=8.6 \mathrm{~Hz}, 2 \mathrm{H}), 6.87(\mathrm{~d}, J=8.7 \mathrm{~Hz}, 2 \mathrm{H}), 5.84$ $(\mathrm{dd}, J=2.8,2.6 \mathrm{~Hz}, 1 \mathrm{H}), 5.32(\mathrm{~d}, J=12.2 \mathrm{~Hz}, 1 \mathrm{H}), 5.32(\mathrm{~s}, 1 \mathrm{H}), 5.18$ $(\mathrm{d}, J=12.2 \mathrm{~Hz}, 1 \mathrm{H}), 3.79(\mathrm{~s}, 3 \mathrm{H}), 3.44(\mathrm{~s}, 3 \mathrm{H}), 2.64-2.30(\mathrm{~m}, 3 \mathrm{H}), 2.05-$ $1.95(\mathrm{~m}, 1 \mathrm{H}) .{ }^{13} \mathrm{C}$ NMR $\left(75 \mathrm{MHz}, \delta \mathrm{ppm} / \mathrm{CDCl}_{3}\right): 170.0(\mathrm{C}), 160.2(\mathrm{C})$, $157.7(\mathrm{C}), 145.0(\mathrm{C}), 135.3(\mathrm{C}), 128.9(2 \mathrm{CH}), 128.9(\mathrm{CH}), 128.4(2 \mathrm{CH})$, $127.8(\mathrm{C}), 127.3(2 \mathrm{CH}), 119.9(\mathrm{CH}), 114.5(2 \mathrm{CH}), 69.9(\mathrm{CH}), 69.0\left(\mathrm{CH}_{2}\right)$, $61.8(\mathrm{C}), 55.6\left(\mathrm{CH}_{3}\right), 34.7\left(\mathrm{CH}_{2}\right), 34.1\left(\mathrm{CH}_{3}\right), 26.3\left(\mathrm{CH}_{2}\right)$. Resonance for $\mathrm{CF}_{3}$ not detected due to a low $\mathrm{S} / \mathrm{N}$ ratio. ${ }^{19} \mathrm{~F}$ NMR $(282 \mathrm{MHz}, \delta \mathrm{ppm} /$ $\left.\mathrm{CDCl}_{3}\right):-74.15(\mathrm{~s}, 3 \mathrm{~F})$.

Triflyl Enol Ether $\mathbf{8} \mathbf{g}_{\mathrm{Me}}$ : Following the general procedure with $\mathbf{7} \mathbf{g}_{\mathbf{M e}}$ (498 mg, $1.57 \mathrm{mmol}), \mathrm{Tf}_{2} \mathrm{NPh}(675 \mathrm{mg}, 1.89 \mathrm{mmol}$ ) and KHMDS $0.5 \mathrm{M}$ in toluene ( $4.8 \mathrm{~mL}, 2.4 \mathrm{mmol}$ ) in $18 \mathrm{~mL}$ of anhydrous THF, and using EtOAc/PE, 4:6 as eluent for the flash chromatography, $\mathbf{8 g}_{\text {Me }}$ was obtained as a white solid (469 mg, $67 \%) . R_{\mathrm{f}}(\mathrm{EtOAC} / \mathrm{PE}, 4: 6)=0.83$. Mp 134.0-135.1 ${ }^{\circ} \mathrm{C}$. HRMS (ESI+): $[\mathrm{M}+\mathrm{H}]^{+}$calcd. for $\mathrm{C}_{18} \mathrm{H}_{20} \mathrm{~N}_{2} \mathrm{O}_{6} \mathrm{SF}_{3}{ }^{+}$ 449.0989, found 449.0990. ${ }^{1} \mathrm{H} \mathrm{NMR}\left(400 \mathrm{MHz}, \delta \mathrm{ppm} / \mathrm{CDCl}_{3}\right)$ : 7.24$7.14(\mathrm{~m}, 3 \mathrm{H}), 7.03-6.99(\mathrm{~m}, 1 \mathrm{H}), 5.83(\mathrm{dd}, J=2.6,2.6 \mathrm{~Hz}, 1 \mathrm{H}), 5.72$ $(\mathrm{s}, 1 \mathrm{H}), 3.85(\mathrm{~s}, 3 \mathrm{H}), 3.49(\mathrm{~s}, 3 \mathrm{H}), 2.70-2.56(\mathrm{~m}, 2 \mathrm{H}), 2.55-2.44(\mathrm{~m}, 1 \mathrm{H})$, $2.31(\mathrm{~s}, 3 \mathrm{H}), 2.25$ (ddd, $J=13.0,7.4,1.9 \mathrm{~Hz}, 1 \mathrm{H}) .{ }^{13} \mathrm{C}$ NMR $(101 \mathrm{MHz}$, $\left.\delta \mathrm{ppm} / \mathrm{CDCl}_{3}\right): 169.9$ (C), 158.1 (C), 145.2 (C), 134.6 (C), 134.2 (C), $131.0(\mathrm{CH}) 128.7(\mathrm{CH}), 126.8(\mathrm{CH}), 125.4(\mathrm{CH}), 120.2,(\mathrm{CH}), 118.1(\mathrm{q}$, $\left.J=322 \mathrm{~Hz}_{1} \mathrm{CF}_{3}\right), 67.3(\mathrm{CH}), 61.4(\mathrm{C}), 54.2\left(\mathrm{CH}_{3}\right), 35.2\left(\mathrm{CH}_{2}\right), 33.9\left(\mathrm{CH}_{3}\right)$, $26.7\left(\mathrm{CH}_{2}\right), 19.5\left(\mathrm{CH}_{3}\right) .{ }^{19} \mathrm{~F} \mathrm{NMR}\left(282 \mathrm{MHz}, \delta \mathrm{ppm} / \mathrm{CDCl}_{3}\right):-74.0(\mathrm{~s}$, $3 \mathrm{~F})$.

Triflyl Enol Ether $\mathbf{8} \mathbf{h}_{\mathrm{Bn}}$ : Following the general procedure with $\mathbf{7} \mathbf{h}_{\mathrm{Bn}}$ $(820 \mathrm{mg}, 2.19 \mathrm{mmol}), \mathrm{Tf}_{2} \mathrm{NPh}(861 \mathrm{mg}, 2.40 \mathrm{mmol})$ and KHMDS $0.5 \mathrm{M}$ 
in toluene ( $6.6 \mathrm{~mL}, 3.3 \mathrm{mmol})$ in $22 \mathrm{~mL}$ of anhydrous THF, and using EtOAc/PE, 2:8 as eluent for the flash chromatography, $\mathbf{8 h}_{\mathbf{B n}}$ was obtained as a white solid (515 mg, $46 \%$ ). $R_{\mathrm{f}}(\mathrm{AcOEt} / \mathrm{PE}, 2: 8)=0.35$. Mp 122.4-122.9 ${ }^{\circ} \mathrm{C}$. HRMS (ESI+): $[\mathrm{M}+\mathrm{H}]^{+}$calcd. for $\mathrm{C}_{20} \mathrm{H}_{22} \mathrm{~F}_{3} \mathrm{~N}_{2} \mathrm{O}_{8} \mathrm{~S}^{+}$ 507.1043, found 507.1042. ${ }^{1} \mathrm{H}$ NMR $\left(300 \mathrm{MHz}, \delta \mathrm{ppm} / \mathrm{CDCl}_{3}\right): 7.45-$ $7.32(\mathrm{~m}, 5 \mathrm{H}), 6.02(\mathrm{dd}, J=2.6,2.6 \mathrm{~Hz}, 1 \mathrm{H}), 5.32(\mathrm{~d}, J=12.0 \mathrm{~Hz}, 1 \mathrm{H})$, $5.19(\mathrm{~d}, J=12.0 \mathrm{~Hz}, 1 \mathrm{H}), 4.90(\mathrm{~s}, 1 \mathrm{H}), 4.30-4.09(\mathrm{~m}, 2 \mathrm{H}), 3.30(\mathrm{~s}, 3 \mathrm{H})$, 2.62-2.44 (m, 2H), 2.29 (ddd, $J=13.1,8.2,7.0 \mathrm{~Hz}, 1 \mathrm{H}$ ), 1.94 (ddd $J=13.2,7.1,3.2 \mathrm{~Hz}, 1 \mathrm{H}), 1.28(\mathrm{t}, J=7.2 \mathrm{~Hz}, 3 \mathrm{H}) .{ }^{13} \mathrm{C} \mathrm{NMR}(75 \mathrm{MHz}$ $\left.\delta \mathrm{ppm} / \mathrm{CDCl}_{3}\right): 170.4(\mathrm{C}), 167.1$ (C), 156.8 (C), 143.4 (C), 135.0 (C), $129.0(\mathrm{CH}), 128.9(2 \mathrm{CH}), 128.6(2 \mathrm{CH}), 121.3(\mathrm{CH}), 69.3\left(\mathrm{CH}_{2}\right), 67.5$ $(\mathrm{CH}), 62.6\left(\mathrm{CH}_{2}\right), 59.8(\mathrm{C}), 34.7\left(\mathrm{CH}_{3}\right), 33.5\left(\mathrm{CH}_{2}\right), 26.3\left(\mathrm{CH}_{2}\right), 13.9$ $\left(\mathrm{CH}_{3}\right)$. Resonance for $\mathrm{CF}_{3}$ not detected due to a low $\mathrm{S} / \mathrm{N}$ ratio. ${ }^{19} \mathrm{~F}$ NMR $\left(282 \mathrm{MHz}, \delta \mathrm{ppm} / \mathrm{CDCl}_{3}\right):-73.51$ (s, 3F).

Triflyl Enol Ether $\mathbf{8 i}_{\mathbf{B n}}$ : Following the general procedure with $\mathbf{7} \mathbf{i}_{\mathbf{B n}}$ (1100 mg, $2.52 \mathrm{mmol}$ ), Tf $2 \mathrm{NPh}(1152 \mathrm{mg}, 3.22 \mathrm{mmol}$ ) and KHMDS $0.5 \mathrm{M}$ in toluene $(7.6 \mathrm{~mL}, 3.8 \mathrm{mmol})$ in $25 \mathrm{~mL}$ of anhydrous THF, and using EtOAc/PE, 2:8 as eluent for the flash chromatography, $\mathbf{8 i}_{\mathbf{B n}}$ was obtained as a white solid (584 mg, $41 \%) . R_{\mathrm{f}}($ AcOEt/PE, 2:8) = 0.40. HRMS (ESI+): $[\mathrm{M}+\mathrm{H}]^{+}$calcd. for $\mathrm{C}_{25} \mathrm{H}_{24} \mathrm{~F}_{3} \mathrm{~N}_{2} \mathrm{O}_{8} \mathrm{~S}^{+} 569.1200$, found 569.1200. ${ }^{1} \mathrm{H}$ NMR (300 MHz, $\left.\delta \mathrm{ppm} / \mathrm{CDCl}_{3}\right): 7.42-7.28(\mathrm{~m}$, $10 \mathrm{H}), 6.04(\mathrm{dd}, J=2.7,2.7 \mathrm{~Hz}, 1 \mathrm{H}), 5.30(\mathrm{~d}, J=12.0 \mathrm{~Hz}, 1 \mathrm{H}), 5.21(\mathrm{~d}$, $J=8.8 \mathrm{~Hz}, 1 \mathrm{H}), 5.18(\mathrm{~d}, J=8.8 \mathrm{~Hz}, 1 \mathrm{H}), 5.09(\mathrm{~d}, J=12.0 \mathrm{~Hz}, 1 \mathrm{H})$, $4.97(\mathrm{~s}, 1 \mathrm{H}), 3.20(\mathrm{~s}, 3 \mathrm{H}), 2.55-2.44(\mathrm{~m}, 2 \mathrm{H}), 2.24-2.36(\mathrm{~m}, 1 \mathrm{H}), 1.95$ (ddd, $J=13.4,6.9,3.7 \mathrm{~Hz}, 1 \mathrm{H}) .{ }^{13} \mathrm{C} \mathrm{NMR}\left(75 \mathrm{MHz}, \delta \mathrm{ppm} / \mathrm{CDCl}_{3}\right.$ ): $170.3(\mathrm{C}), 166.9(\mathrm{C}), 156.7(\mathrm{C}), 143.4(\mathrm{C}), 135.0(\mathrm{C}), 134.5(\mathrm{C}), 129.0$ $(\mathrm{CH}), 128.9(2 \mathrm{CH}), 128.8(2 \mathrm{CH}), 128.7(2 \mathrm{CH}), 128.5(2 \mathrm{CH}), 127.0(\mathrm{CH})$ $121.2(\mathrm{CH}), 118.5\left(\mathrm{q}, J=322 \mathrm{~Hz}, \mathrm{CF}_{3}\right), 69.2\left(\mathrm{CH}_{2}\right), 68.4\left(\mathrm{CH}_{2}\right), 67.5$ $(\mathrm{CH}), 59.9(\mathrm{C}), 34.6\left(\mathrm{CH}_{3}\right), 33.4\left(\mathrm{CH}_{2}\right), 26.3\left(\mathrm{CH}_{2}\right) .{ }^{19} \mathrm{~F} \mathrm{NMR}(282 \mathrm{MHz}$, $\left.\delta \mathrm{ppm} / \mathrm{CDCl}_{3}\right):-73.33$.

Triflyl Enol Ether $\mathbf{8}_{\mathbf{j}_{\mathbf{B n}}}$ : Following the general procedure with $\mathbf{7} \mathbf{j}_{\mathbf{B} \mathbf{n}}$ (709 mg, $1.81 \mathrm{mmol}), \mathrm{Tf}_{2} \mathrm{NPh}(826 \mathrm{mg}, 2.31 \mathrm{mmol})$ and KHMDS $0.5 \mathrm{M}$ in toluene (5.4 mL, $2.7 \mathrm{mmol}$ ) in $25 \mathrm{~mL}$ of anhydrous THF, and using EtOAc/PE, 2:8 as eluent for the flash chromatography, $\mathbf{8 j}_{\mathbf{B n}}$ was obtained as a white solid (800 mg, $84 \%) . R_{\mathrm{f}}(\mathrm{EtOAc} / \mathrm{PE}, 2: 8)=0.49$ HRMS (ESI+): $[\mathrm{M}+\mathrm{H}]^{+}$calcd. for $\mathrm{C}_{24} \mathrm{H}_{24} \mathrm{~F}_{3} \mathrm{~N}_{2} \mathrm{O}_{6} \mathrm{~S}^{+}$525.1302, found 525.1302. Mp 103.3-103.7 ${ }^{\circ} \mathrm{C} .{ }^{1} \mathrm{H}$ NMR $\left(300 \mathrm{MHz}, \delta \mathrm{ppm} / \mathrm{CDCl}_{3}\right)$ : 7.35-7.24 (m, 6H), 7.20-7.15 (m, 2H), 7.08-7.03 (m, 2H) $6.02(\mathrm{dd}, J=$ 2.5, $2.5 \mathrm{~Hz}, 1 \mathrm{H}), 5.06-4.98(\mathrm{~m}, 2 \mathrm{H}), 4.58(\mathrm{dd}, J=11.7,3.8 \mathrm{~Hz}, 1 \mathrm{H})$, $3.30(\mathrm{~s}, 3 \mathrm{H}), 2.91-2.68(\mathrm{~m}, 2 \mathrm{H}), 2.49-2.41(\mathrm{~m}, 2 \mathrm{H}), 2.30-2.17(\mathrm{~m}, 1 \mathrm{H})$, 1.86 (ddd, $J=13.1,6.4,3.1 \mathrm{~Hz}, 1 \mathrm{H}) .{ }^{13} \mathrm{C} \mathrm{NMR}(75 \mathrm{MHz}, \delta \mathrm{ppm} /$ $\left.\mathrm{CDCl}_{3}\right)$ : 170.6 (C), 157.7 (C), 145.0 (C), 136.8 (C), 135.1 (C), 129.4 $(2 \mathrm{CH}), 128.7(2 \mathrm{CH}), 128.6(\mathrm{CH}), 128.5(2 \mathrm{CH}), 128.0(2 \mathrm{CH}), 126.9(\mathrm{CH})$ $121.3(\mathrm{CH}), 118.5\left(\mathrm{q}, J=320 \mathrm{~Hz}, \mathrm{CF}_{3}\right), 69.3(\mathrm{CH}), 68.6\left(\mathrm{CH}_{2}\right), 60.0(\mathrm{C})$ $36.6\left(\mathrm{CH}_{2}\right), 34.1\left(\mathrm{CH}_{3}\right), 33.3\left(\mathrm{CH}_{2}\right), 26.2\left(\mathrm{CH}_{2}\right) .{ }^{19} \mathrm{~F} \mathrm{NMR}(282 \mathrm{MHz}, \delta$ $\left.\mathrm{ppm} / \mathrm{CDCl}_{3}\right):-73.7(\mathrm{~s}, 3 \mathrm{~F})$.

General Procedure for Olefins 9: To a solution of $\mathbf{8}$ in anhydrous DMF (ca $0.1 \mathrm{M}$ ) under an argon atmosphere was added palladium diacetate (20-50 mol-\%), triphenylphosphane (60-100 mol-\%), formic acid ( 2 equiv.) and tributylamine ( 3 equiv.). The resulting mixture was heated at $80{ }^{\circ} \mathrm{C}$ and stirred at this temperature for $4 \mathrm{~h}$. After cooling to room temperature, the mixture was diluted with ethyl acetate, washed twice $1 \mathrm{M} \mathrm{HCl}$ and twice with brine. The organic layer was dried with anhydrous sodium sulfate, filtered and concentrated in vacuo to afford the crude product, which was purified by flash chromatography.

Olefin $\mathbf{9} \mathbf{h}_{\mathbf{B n}}$ : Following the general procedure with $\mathbf{8 h}_{\mathbf{B n}}(400 \mathrm{mg}$ $0.79 \mathrm{mmol}), \mathrm{Pd}(\mathrm{OAc})_{2}(89 \mathrm{mg}, 0.40 \mathrm{mmol}), \mathrm{PPh}_{3}(207 \mathrm{mg}$, $0.79 \mathrm{mmol})$, formic acid $(60 \mu \mathrm{L}, 1.59 \mathrm{mmol})$ and $n \mathrm{Bu}_{3} \mathrm{~N}(565 \mu \mathrm{L}$, $2.38 \mathrm{mmol})$ in anhydrous DMF (16 mL), and using EtOAc/PE, 2:8 as eluent for the flash chromatography, $\mathbf{9} \mathbf{h}_{\mathbf{B}}$ was obtained as a colorless oil (161 mg, $57 \%)$. $R_{\mathrm{f}}(\mathrm{EtOAC} / \mathrm{PE}, 2: 8)=0.27$. HRMS (ESI+):
$[\mathrm{M}+\mathrm{H}]^{+}$calcd. for $\mathrm{C}_{19} \mathrm{H}_{23} \mathrm{~N}_{2} \mathrm{O}_{5}{ }^{+} 359.1601$, found 359.1604. ${ }^{1} \mathrm{H}$ NMR (300 MHz, $\left.\delta ~ p p m / \mathrm{CDCl}_{3}\right): 7.47-7.30(\mathrm{~m}, 5 \mathrm{H}), 6.19-6.13(\mathrm{~m}, 1 \mathrm{H}), 5.34-$ $5.28(\mathrm{~m}, 1 \mathrm{H}), 5.30(\mathrm{~d}, \mathrm{~J}=12.1 \mathrm{~Hz}, 1 \mathrm{H}), 5.20(\mathrm{~d}, \mathrm{~J}=12.1 \mathrm{~Hz}, 1 \mathrm{H}), 4.76$ $(\mathrm{s}, 1 \mathrm{H}), 4.25-4.13(\mathrm{~m}, 2 \mathrm{H}), 3.28(\mathrm{~s}, 3 \mathrm{H}), 2.52-2.42(\mathrm{~m}, 2 \mathrm{H}), 2.20-2.06$ $(\mathrm{m}, 1 \mathrm{H}), 1.95-1.84(\mathrm{~m}, 1 \mathrm{H}), 1.26(\mathrm{t}, \mathrm{J}=7.1 \mathrm{~Hz}, 3 \mathrm{H}) .{ }^{13} \mathrm{C} \mathrm{NMR}(75 \mathrm{MHz}$, $\left.\delta \mathrm{ppm} / \mathrm{CDCl}_{3}\right): 175.1(\mathrm{C}), 168.1(\mathrm{C}), 156.9(\mathrm{C}), 139.2(\mathrm{CH}), 135.3(\mathrm{C})$, $128.8(2 \mathrm{CH}), 128.8(\mathrm{CH}), 128.3(2 \mathrm{CH}), 126.5(\mathrm{CH}), 69.2(\mathrm{CH}), 68.9$ $\left(\mathrm{CH}_{2}\right), 61.7\left(\mathrm{CH}_{2}\right), 60.9(\mathrm{C}), 34.8\left(\mathrm{CH}_{3}\right), 34.8\left(\mathrm{CH}_{2}\right), 31.8\left(\mathrm{CH}_{2}\right), 14.2$ $\left(\mathrm{CH}_{3}\right)$.

Olefin $\mathbf{9 i}_{\mathbf{B n}}$ : Following the general procedure with $\mathbf{8 i}_{\mathbf{B n}}(584 \mathrm{mg}$, $1.03 \mathrm{mmol}), \mathrm{Pd}(\mathrm{OAc})_{2}(46 \mathrm{mg}, 0.20 \mathrm{mmol}), \mathrm{PPh}_{3}(162 \mathrm{mg}$, $0.62 \mathrm{mmol})$, formic acid $(77 \mu \mathrm{L}, 2.04 \mathrm{mmol})$ and $n \mathrm{Bu}_{3} \mathrm{~N}(734 \mu \mathrm{L}$, $3.09 \mathrm{mmol})$ in anhydrous DMF (10 mL), and using EtOAc/PE, 2:8 as eluent for the flash chromatography, $\mathbf{9 i}_{\mathbf{B n}}$ was obtained as a yellowish oil (332 mg, $77 \%) . R_{\mathrm{f}}(\mathrm{AcOEt} / \mathrm{PE}, 3: 7)=0.64$. HRMS (ESI+): $[\mathrm{M}+\mathrm{H}]^{+}$ calcd. for $\mathrm{C}_{24} \mathrm{H}_{25} \mathrm{~N}_{2} \mathrm{O}_{5}+421.1758$, found 421.1758 . ${ }^{1} \mathrm{H}$ NMR $(300 \mathrm{MHz}$, $\left.\delta \mathrm{ppm} / \mathrm{CDCl}_{3}\right): 7.42-7.26(\mathrm{~m}, 10 \mathrm{H}), 6.09(\mathrm{ddd}, \mathrm{J}=5.8,2.4,2.4 \mathrm{~Hz}$, $1 \mathrm{H}), 5.30(\mathrm{~d}, J=12.0 \mathrm{~Hz}, 1 \mathrm{H}), 5.23-5.09(\mathrm{~m}, 4 \mathrm{H}), 4.82(\mathrm{~s}, 1 \mathrm{H}), 3.23(\mathrm{~s}$, $3 \mathrm{H}$ ), 2.45 (dddd, J = 7.1, 4.6, 2.4, $2.4 \mathrm{~Hz}, 2 \mathrm{H}$ ), 2.12 (ddd, J = 13.1, 8.6, $7.1 \mathrm{~Hz}, 1 \mathrm{H}), 1.89$ (ddd, J = 13.2, 6.7, 4.6 Hz, 1H). ${ }^{13} \mathrm{C} \mathrm{NMR}(75 \mathrm{MHz}$, $\delta \mathrm{ppm} / \mathrm{CDCl}_{3}$ ): $174.9(\mathrm{C}), 168.0(\mathrm{C}), 156.8(\mathrm{C}), 139.3(\mathrm{CH}), 135.3(\mathrm{C})$, $134.8(\mathrm{C}), 128.9(2 \mathrm{CH}), 128.8(2 \mathrm{CH}), 128.8(\mathrm{CH}), 128.7(2 \mathrm{CH}), 128.7$ $(\mathrm{CH}), 128.4(2 \mathrm{CH}), 126.5(\mathrm{CH}), 69.1(\mathrm{CH}), 69.0\left(\mathrm{CH}_{2}\right), 67.5\left(\mathrm{CH}_{2}\right), 61.0$ (C), $34.8\left(\mathrm{CH}_{2}\right), 34.8\left(\mathrm{CH}_{3}\right), 31.8\left(\mathrm{CH}_{2}\right)$.

Olefin $\mathbf{9 j}_{\mathbf{B n}}$ : Following the general procedure with $\mathbf{8}_{\mathbf{B n}}$ (597 $\mathrm{mg}$, $1.14 \mathrm{mmol}), \mathrm{Pd}(\mathrm{OAc})_{2}(51 \mathrm{mg}, 0.23 \mathrm{mmol}), \mathrm{PPh}_{3}(179 \mathrm{mg}$, $0.68 \mathrm{mmol})$, formic acid $(81 \mu \mathrm{L}, 2.15 \mathrm{mmol})$ and $n \mathrm{Bu}_{3} \mathrm{~N}(860 \mu \mathrm{L}$, $3.62 \mathrm{mmol})$ in anhydrous DMF (23 mL), and using EtOAc/PE, 2:8 as eluent for the flash chromatography, $\mathbf{9 j}_{\mathbf{B n}}$ was obtained as a yellowish oil $(380 \mathrm{mg}, 89 \%) . R_{\mathrm{f}}(\mathrm{AcOEt} / \mathrm{PE}, 2: 8)=0.26$. HRMS (ESI+): $[\mathrm{M}+\mathrm{H}]^{+}$ calcd. for $\mathrm{C}_{23} \mathrm{H}_{25} \mathrm{~N}_{2} \mathrm{O}_{3}{ }^{+} 377.1860$, found 377.1860. ${ }^{1} \mathrm{H}$ NMR (300 MHz, $\left.\delta \mathrm{ppm} / \mathrm{CDCl}_{3}\right): 7.38-7.30(\mathrm{~m}, 3 \mathrm{H}), 7.30-7.23(\mathrm{~m}, 3 \mathrm{H}), 7.21-7.14(\mathrm{~m}$, $2 \mathrm{H}), 7.14-7.07(\mathrm{~m}, 2 \mathrm{H}), 6.23-6.15(\mathrm{~m}, 1 \mathrm{H}), 5.68-5.61(\mathrm{~m}, 1 \mathrm{H}), 5.06$ $(\mathrm{dd}, J=14.5,12.7,3 \mathrm{H}), 4.46(\mathrm{dd}, J=11.4,3.4 \mathrm{~Hz}, 1 \mathrm{H}), 3.27(\mathrm{~s}, 3 \mathrm{H})$, $2.84(\mathrm{dd}, J=14.0,3.5 \mathrm{~Hz}, 1 \mathrm{H}), 2.59(\mathrm{dd}, J=13.8,11.8 \mathrm{~Hz}, 1 \mathrm{H}), 2.51-$ $2.40(\mathrm{~m}, 2 \mathrm{H}), 2.16-2.01(\mathrm{~m}, 1 \mathrm{H}), 1.94-1.81(\mathrm{~m}, 1 \mathrm{H}) .{ }^{13} \mathrm{C} \mathrm{NMR}(75 \mathrm{MHz}$, $\left.\delta \mathrm{ppm} / \mathrm{CDCl}_{3}\right): 175.5(\mathrm{C}), 158.0(\mathrm{C}), 138.5(\mathrm{CH}), 137.5(\mathrm{C}), 135.5(\mathrm{C})$, $129.4(2 \mathrm{CH}), 128.6(2 \mathrm{CH}), 128.4(2 \mathrm{CH}), 128.4(\mathrm{CH}), 127.9(2 \mathrm{CH}), 127.1$ $(\mathrm{CH}), 126.7(\mathrm{CH}), 70.6,(\mathrm{CH}), 68.2\left(\mathrm{CH}_{2}\right), 62.0(\mathrm{C}), 36.8\left(\mathrm{CH}_{2}\right), 34.3$ $\left(\mathrm{CH}_{2}\right), 33.9\left(\mathrm{CH}_{3}\right), 31.7\left(\mathrm{CH}_{2}\right)$.

General Procedure for Tetracyclic Compounds 10: To a solution of 8 in anhydrous DMF (ca $0.1 \mathrm{M}$ ) under an argon atmosphere was added palladium diacetate (20-50 mol-\%), triphenylphosphane (60$100 \mathrm{~mol}-\%)$ and tributylamine (3 equiv.). The resulting mixture was heated at $80{ }^{\circ} \mathrm{C}$ and stirred at this temperature for 1-23 h. After cooling to room temperature, the mixture was diluted with ethyl acetate, washed twice $1 \mathrm{M} \mathrm{HCl}$ and twice with brine. The organic layer was dried with anhydrous sodium sulfate, filtered and concentrated in vacuo to afford the crude product, which was purified by flash chromatography.

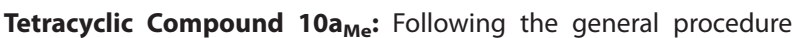
with $8 \mathrm{a}_{\mathrm{Me}}(494 \mathrm{mg}, 1.14 \mathrm{mmol}), \mathrm{Pd}(\mathrm{OAc})_{2}(128 \mathrm{mg}, 0.57 \mathrm{mmol})$, $\mathrm{PPh}_{3}(298 \mathrm{mg}, 1.14 \mathrm{mmol})$ and $n \mathrm{Bu}_{3} \mathrm{~N}(810 \mu \mathrm{L}, 3.41 \mathrm{mmol})$ in anhydrous DMF (11 mL) for $1.5 \mathrm{~h}$, and using EtOAc/PE, 4:6 as eluent for the flash chromatography, $10 \mathbf{a}_{\mathrm{Me}}$ was obtained as a yellow solid (321 mg, $99 \%$ \%). $R_{\mathrm{f}}(\mathrm{EtOAC} / \mathrm{PE}, 4: 6)=0.53$. Mp 152.0-154.0 ${ }^{\circ} \mathrm{C}$. HRMS (ESI+): $[\mathrm{M}+\mathrm{H}]^{+}$calcd. for $\mathrm{C}_{16} \mathrm{H}_{17} \mathrm{~N}_{2} \mathrm{O}_{3}{ }^{+} 285.1234$, found $285.1235 .{ }^{1} \mathrm{H}$ NMR (300 MHz, $\left.\delta p p m / \mathrm{CDCl}_{3}\right)$ : 7.50-7.41 (m, 2H), 7.38-7.26 (m, 2H), $6.14(\mathrm{dd}, \mathrm{J}=3.4,2.0 \mathrm{~Hz}, 1 \mathrm{H}), 5.43(\mathrm{~s}, 1 \mathrm{H}), 3.92(\mathrm{~s}, 3 \mathrm{H}), 3.35-3.21(\mathrm{~m}$, $1 \mathrm{H}), 3.10(\mathrm{~s}, 3 \mathrm{H}), 2.73(\mathrm{ddd}, \mathrm{J}=12.4,8.7,3.5 \mathrm{~Hz}, 1 \mathrm{H}), 2.37(\mathrm{dd}, \mathrm{J}=$ $12.4,6.5 \mathrm{~Hz}, 1 \mathrm{H}), 2.12-2.02(\mathrm{~m}, 1 \mathrm{H}) .{ }^{13} \mathrm{C} \mathrm{NMR}(75 \mathrm{MHz}, \delta \mathrm{ppm} /$ $\left.\mathrm{CDCl}_{3}\right):=173.8(\mathrm{C}), 158.4$ (C), 147.0 (C), 146.1 (C), 135.5 (C), 129.6 
$(\mathrm{CH}), 128.9(\mathrm{CH}), 126.0(\mathrm{CH}), 123.2(\mathrm{CH}), 122.4(\mathrm{CH}), 68.3(\mathrm{CH}), 66.9$ (C), $54.0\left(\mathrm{CH}_{3}\right), 37.6\left(\mathrm{CH}_{2}\right), 34.3\left(\mathrm{CH}_{3}\right), 33.2\left(\mathrm{CH}_{2}\right)$.

Tetracyclic Compound $\mathbf{1 0 d}_{\mathrm{Me}}$ : Following the general procedure with $8 \mathbf{d}_{\mathrm{Me}}(425 \mathrm{mg}, 0.89 \mathrm{mmol}), \mathrm{Pd}(\mathrm{OAc})_{2}(100 \mathrm{mg}, 0.45 \mathrm{mmol})$ $\mathrm{PPh}_{3}(233 \mathrm{mg}, 0.89 \mathrm{mmol})$ and $n \mathrm{Bu}_{3} \mathrm{~N}(630 \mu \mathrm{L}, 2.65 \mathrm{mmol})$ in anhydrous DMF ( $9 \mathrm{~mL}$ ) for $4 \mathrm{~h}$, and using EtOAc/PE, 5:5 as eluent for the flash chromatography, $\mathbf{1 0 a _ { M e }}$ was obtained as a yellow solid (288 mg, $98 \%$ \%). $R_{\mathrm{f}}(\mathrm{EtOAc} / \mathrm{PE}, 5: 5)=0.46 . \mathrm{Mp} 189.0-191.0{ }^{\circ} \mathrm{C}$. HRMS (ESI+): $[\mathrm{M}+\mathrm{H}]^{+}$calcd. for $\mathrm{C}_{16} \mathrm{H}_{16} \mathrm{~N}_{3} \mathrm{O}_{5}{ }^{+} 330.1084$, found $330.1086 .{ }^{1} \mathrm{H}$ NMR $\left(300 \mathrm{MHz}, \delta \mathrm{ppm} / \mathrm{CDCl}_{3}\right): 8.25(\mathrm{~d}, J=2.0 \mathrm{~Hz}, 1 \mathrm{H}), 8.16(\mathrm{dd}, \mathrm{J}=$ 8.4, $2.2 \mathrm{~Hz}, 1 \mathrm{H}), 7.63(\mathrm{~d}, \mathrm{~J}=8.4 \mathrm{~Hz}, 1 \mathrm{H}), 6.35(\mathrm{dd}, \mathrm{J}=3.4,2.0 \mathrm{~Hz}$ $2 \mathrm{H}), 5.50(\mathrm{~s}, 1 \mathrm{H}), 3.94(\mathrm{~s}, 3 \mathrm{H}), 3.40-3.26(\mathrm{~m}, 1 \mathrm{H}), 3.11(\mathrm{~s}, 3 \mathrm{H}), 2.80$ (ddd, J = 14.9, 8.7, 3.6 Hz, 1H), $2.42(\mathrm{dd}, \mathrm{J}=12.5,6.5 \mathrm{~Hz}, 1 \mathrm{H}), 2.18-$ $2.08(\mathrm{~m}, 1 \mathrm{H}) .{ }^{13} \mathrm{C}$ NMR $\left(75 \mathrm{MHz}\right.$, $\left.\delta \mathrm{ppm} / \mathrm{CDCl}_{3}\right): 172.7(\mathrm{C}), 158.3(\mathrm{C})$ $152.5(\mathrm{C}), 149.5(\mathrm{C}), 144.9(\mathrm{C}), 136.9(\mathrm{C}), 127.1(\mathrm{CH}), 126.9(\mathrm{CH}), 123.9$ $(\mathrm{CH}), 117.5(\mathrm{CH}), 67.9(\mathrm{CH}), 67.3(\mathrm{C}), 54.3\left(\mathrm{CH}_{3}\right), 37.8\left(\mathrm{CH}_{2}\right), 34.3\left(\mathrm{CH}_{3}\right)$, $33.4\left(\mathrm{CH}_{2}\right)$.

Tetracyclic Compound $10 \mathrm{f}_{\mathrm{Bn}}$ : Following the general procedure with $8 \mathbf{f}_{\mathrm{Bn}}(550 \mathrm{mg}, 1.02 \mathrm{mmol}), \mathrm{Pd}(\mathrm{OAc})_{2}(46 \mathrm{mg}, 0.20 \mathrm{mmol}), \mathrm{PPh}_{3}$ $(160 \mathrm{mg}, 0.61 \mathrm{mmol})$ and $n \mathrm{Bu}_{3} \mathrm{~N}(727 \mu \mathrm{L}, 3.06 \mathrm{mmol})$ in anhydrous DMF $(20 \mathrm{~mL})$ for $4 \mathrm{~h}$, and using EtOAc/PE, 5:5 as eluent for the flash chromatography, $\mathbf{1 0 f}_{\mathbf{B n}}$ was obtained as a white solid (388 $\mathrm{mg}$, $98 \%) . R_{f}(\mathrm{AcOEt} / \mathrm{PE}, 2: 8)=0.14$. Mp 122.3-124.3 ${ }^{\circ} \mathrm{C}$. HRMS (ESI+): $[\mathrm{M}+\mathrm{H}]^{+}$calcd. for $\mathrm{C}_{23} \mathrm{H}_{23} \mathrm{~N}_{2} \mathrm{O}_{4}{ }^{+}$391.1652, found 391.1654. ${ }^{1} \mathrm{H}$ NMR (300 MHz, $\left.\delta \mathrm{ppm} / \mathrm{CDCl}_{3}\right): 7.43-7.37(\mathrm{~m}, 5 \mathrm{H}), 7.34(\mathrm{~d}, \mathrm{~J}=8.4 \mathrm{~Hz}, 1 \mathrm{H})$ $6.92(\mathrm{~d}, J=2.5 \mathrm{~Hz}, 1 \mathrm{H}), 6.84(\mathrm{dd}, J=8.4,2.5 \mathrm{~Hz}, 1 \mathrm{H}), 6.11(\mathrm{dd}, \mathrm{J}=$ 3.5, $2.0 \mathrm{~Hz}, 1 \mathrm{H}), 5.40(\mathrm{~s}, 1 \mathrm{H}), 5.35(\mathrm{~d}, J=12.1 \mathrm{~Hz}, 1 \mathrm{H}), 5.29(\mathrm{~d}, J=$ $12.1 \mathrm{~Hz}, 1 \mathrm{H}), 3.81(\mathrm{~s}, 3 \mathrm{H}), 3.19-3.33(\mathrm{~m}, 1 \mathrm{H}), 2.71(\mathrm{ddd}, \mathrm{J}=16.6,8.1$, $3.5 \mathrm{~Hz}, 1 \mathrm{H}), 2.32(\mathrm{dd}, \mathrm{J}=12.5,6.6 \mathrm{~Hz}, 1 \mathrm{H}), 2.02(\mathrm{ddd}, \mathrm{J}=12.5,9.4 \mathrm{~Hz}$ 1H). $\left.{ }^{13} \mathrm{C} \mathrm{NMR} \mathrm{(75} \mathrm{MHz,} \delta \mathrm{ppm} / \mathrm{CDCl}_{3}\right): 173.9$ (C), 161.1 (C), 157.7 (C), 147.0 (C), $138.1(\mathrm{C}), 136.8(\mathrm{C}), 135.6(\mathrm{C}), 128.9(2 \mathrm{CH}), 128.7(\mathrm{CH})$, $128.2(2 \mathrm{CH}), 126.8(\mathrm{CH}), 123.1(\mathrm{CH}), 116.3(\mathrm{CH}), 106.3(\mathrm{CH}), 68.7$ $\left(\mathrm{CH}_{2}\right), 68.0(\mathrm{CH}), 67.5(\mathrm{C}), 55.7\left(\mathrm{CH}_{3}\right), 37.5\left(\mathrm{CH}_{2}\right), 34.4\left(\mathrm{CH}_{3}\right), 33.0$ $\left(\mathrm{CH}_{2}\right)$.

Tetracyclic Compound $\mathbf{1 0 g}_{\mathrm{Me}}$ : Following the general procedure with $\mathbf{8} \mathbf{g}_{\mathrm{Me}}(400 \mathrm{mg}, 0.89 \mathrm{mmol}), \mathrm{Pd}(\mathrm{OAc})_{2}(100 \mathrm{mg}, 0.44 \mathrm{mmol})$, $\mathrm{PPh}_{3}(234 \mathrm{mg}, 0.89 \mathrm{mmol})$ and $n \mathrm{Bu}_{3} \mathrm{~N}(640 \mu \mathrm{L}, 2.69 \mathrm{mmol})$ in anhydrous DMF ( $9 \mathrm{~mL}$ ) for $23 \mathrm{~h}$, and using EtOAc/PE, 3:7 as eluent for the flash chromatography, $\mathbf{1 0 g}_{\mathrm{Me}}$ was obtained as a yellow solid (246 mg, 93 \%). $R_{\mathrm{f}}(\mathrm{EtOAC} / \mathrm{PE}, 3: 7)=0.44 . \mathrm{Mp} 170.0-170.8^{\circ} \mathrm{C}$. HRMS (ESI+): $[\mathrm{M}+\mathrm{H}]^{+}$calcd. for $\mathrm{C}_{17} \mathrm{H}_{19} \mathrm{~N}_{2} \mathrm{O}_{3}{ }^{+} 299.1390$, found $299.1390 .{ }^{1} \mathrm{H}$ NMR $\left(400 \mathrm{MHz}, \delta \mathrm{ppm} / \mathrm{CDCl}_{3}\right): 7.28(\mathrm{~d}, J=7.6 \mathrm{~Hz}, 1 \mathrm{H}), 7.22(\mathrm{dd}, \mathrm{J}=$ 7.6, $7.3 \mathrm{~Hz}, 1 \mathrm{H}), 7.07(\mathrm{~d}, J=7.3 \mathrm{~Hz}, 1 \mathrm{H}), 6.09(\mathrm{dd}, J=3.3,1.9 \mathrm{~Hz}$ $1 \mathrm{H}), 5.54(\mathrm{~s}, 1 \mathrm{H}), 3.92(\mathrm{~s}, 3 \mathrm{H}), 3.25$ (dddd, $\mathrm{J}=16.5,9.2,6.6,2.0 \mathrm{~Hz}$, 1H), $3.10(\mathrm{~s}, 3 \mathrm{H}), 2.71(\mathrm{ddd}, \mathrm{J}=16.5,8.7,3.5 \mathrm{~Hz}, 1 \mathrm{H}), 2.40(\mathrm{~s}, 3 \mathrm{H})$ $2.36(\mathrm{dd}, \mathrm{J}=12.2,6.6 \mathrm{~Hz}, 1 \mathrm{H}), 2.10-2.01(\mathrm{~m}, 1 \mathrm{H}) .{ }^{13} \mathrm{C} \mathrm{NMR}(101 \mathrm{MHz}$, $\delta \mathrm{ppm} / \mathrm{CDCl}_{3}$ ): 173.9 (C), 158.8 (C), 147.0 (C), 143.0 (C), 137.3 (C), $135.7(\mathrm{C}), 130.5(\mathrm{CH}), 129.7(\mathrm{CH}), 122.7(\mathrm{CH}), 119.9(\mathrm{CH}), 68.9(\mathrm{CH})$, $66.6(\mathrm{C}), 54.0\left(\mathrm{CH}_{3}\right), 37.5\left(\mathrm{CH}_{2}\right), 34.2\left(\mathrm{CH}_{3}\right), 32.9\left(\mathrm{CH}_{2}\right), 18.2\left(\mathrm{CH}_{3}\right)$.

Keto Ester 11: To a solution of phenyl 3-oxobutanoate $(2.50 \mathrm{~g}$ $13 \mathrm{mmol}$ ) in $32 \mathrm{~mL}$ of anhydrous DMF was added $\mathrm{K}_{2} \mathrm{CO}_{3}(3.59 \mathrm{~g}$ $26 \mathrm{mmol})$, 1,4-dibromobutane $(4.7 \mathrm{~mL}, 39 \mathrm{mmol})$ and $n \mathrm{Bu}_{4} \mathrm{Nl}$ (480 $\mathrm{mg}, 1.3 \mathrm{mmol})$, and the resulting mixture was stirred at room temperature for 2 days. Brine $(30 \mathrm{~mL})$ and ethyl acetate $(30 \mathrm{~mL})$ were added and the organic layer separated. The aqueous layer was extracted twice with EtOAc and the combined organic layers were dried with anhydrous $\mathrm{Na}_{2} \mathrm{SO}_{4}$, concentrated in vacuo and purified by flash chromatography eluted with EtOAC/PE, 1:9 to afford $\mathbf{1 1}$ as a colorless oil $(1.46 \mathrm{~g}, 46 \%) . R_{\mathrm{f}}(\mathrm{ACOEt} / \mathrm{PE}, 1: 9)=0.40 .{ }^{1} \mathrm{H}$ NMR $\left(300 \mathrm{MHz}, \delta \mathrm{ppm} / \mathrm{CDCl}_{3}\right): 7.85(\mathrm{dd}, \mathrm{J}=8.3,1.4 \mathrm{~Hz}, 2 \mathrm{H}), 7.55-7.45$ $(\mathrm{m}, 1 \mathrm{H}), 7.44-7.35(\mathrm{~m}, 2 \mathrm{H}), 4.04(\mathrm{q}, \mathrm{J}=7.1 \mathrm{~Hz}, 2 \mathrm{H}), 2.49-2.21(\mathrm{~m}$, $4 \mathrm{H}), 1.86-1.60(\mathrm{~m}, 4 \mathrm{H}), 0.96(\mathrm{t}, J=7.1 \mathrm{~Hz}, 3 \mathrm{H}) .{ }^{13} \mathrm{C} \mathrm{NMR}(75 \mathrm{MHz}$, $\left.\delta \mathrm{ppm} / \mathrm{CDCl}_{3}\right): 196.2(\mathrm{C}), 174.8(\mathrm{C}), 135.56(\mathrm{C}), 132.7(\mathrm{CH}), 128.9$ $(2 \mathrm{CH}), 128.5(2 \mathrm{CH}), 63.8(\mathrm{C}), 61.37\left(\mathrm{CH}_{2}\right), 35.2\left(2 \mathrm{CH}_{2}\right), 26.4\left(2 \mathrm{CH}_{2}\right)$, $13.8\left(\mathrm{CH}_{3}\right)$.

Acylhydrazone 12: Keto ester 11 (350 mg, $1.42 \mathrm{mmol})$ and methylhydrazine $(230 \mu \mathrm{L}, 4.39 \mathrm{mmol})$ were allowed to react neat at room temperature for 3 days. Purification of the resulting material by flash chromatography eluted with EtOAc/PE, 2:8 afforded 12 (320 mg, $99 \%$ ) as a white solid. $R_{\mathrm{f}}(\mathrm{AcOEt} / \mathrm{PE}, 2: 8)=0.50 . \mathrm{Mp} 89.8-91.2{ }^{\circ} \mathrm{C}$. HRMS (ESI+): $[\mathrm{M}+\mathrm{H}]^{+}$calcd. for ${ }_{14} \mathrm{H}_{17} \mathrm{~N}_{2} \mathrm{O}^{+}$229.1335, found 229.1337. ${ }^{1} \mathrm{H}$ NMR $\left(300 \mathrm{MHz}, \delta \mathrm{ppm} / \mathrm{CDCl}_{3}\right)$ : 7.77-7.71 (m, 2H), 7.43$7.38(\mathrm{~m}, 3 \mathrm{H}), 3.32(\mathrm{~s}, 3 \mathrm{H}), 2.21-1.90(\mathrm{~m}, 8 \mathrm{H}) .{ }^{13} \mathrm{C}$ NMR $(75 \mathrm{MHz}, \delta$ $\left.\mathrm{ppm} / \mathrm{CDCl}_{3}\right): 180.8(\mathrm{C}), 161.3(\mathrm{C}), 130.7(\mathrm{C}), 129.9(\mathrm{CH}), 128.9(2 \mathrm{CH})$, $126.3(2 \mathrm{CH}), 56.9(\mathrm{C}), 36.0\left(2 \mathrm{CH}_{2}\right), 31.5\left(\mathrm{CH}_{3}\right), 27.5\left(2 \mathrm{CH}_{2}\right)$.

Spirobicyclic Pyrazolidinone 3b: To a solution of 12 (243 mg, $1.06 \mathrm{mmol})$ in methanol $(18 \mathrm{~mL})$ was added $10 \% \mathrm{Pd} / \mathrm{C}(113 \mathrm{mg})$ and the solution was placed under an atmosphere of hydrogen with vigorous stirring for three days at room temperature. The resulting suspension was filtered through celite, concentrated in vacuo and purified by flash chromatography eluted with $\mathrm{EtOAc} / \mathrm{CH}_{2} \mathrm{Cl}_{2}, 4: 6$ to afford pure $\mathbf{3 b}$ (143 mg, $59 \%)$ as a white solid. $R_{\mathrm{f}}\left(\mathrm{AcOEt} / \mathrm{CH}_{2} \mathrm{Cl}_{2}\right.$, 4:6) $=0.24$. HRMS (ESI+): $[\mathrm{M}+\mathrm{H}]^{+}$calcd. for $\mathrm{C}_{14} \mathrm{H}_{19} \mathrm{~N}_{2} \mathrm{O}^{+} 231.1492$, found 231.1491. ${ }^{1} \mathrm{H}$ NMR $\left(300 \mathrm{MHz}, \delta \mathrm{ppm} / \mathrm{CDCl}_{3}\right): 7.39-7.29(\mathrm{~m}$, $3 \mathrm{H}), 7.23-7.18(\mathrm{~m}, 2 \mathrm{H}), 4.73$ (broad s, 1H), $4.31(\mathrm{~s}, 1 \mathrm{H}), 3.15(\mathrm{~s}, 3 \mathrm{H})$, 2.17-2.04 $(\mathrm{m}, 1 \mathrm{H}), 1.89-1.75(\mathrm{~m}, 2 \mathrm{H}), 1.72-1.55(\mathrm{~m}, 3 \mathrm{H}), 1.42-1.31$ $(\mathrm{m}, 1 \mathrm{H}), 1.29-1.15(\mathrm{~m}, 1 \mathrm{H}) .{ }^{13} \mathrm{C} \mathrm{NMR}\left(75 \mathrm{MHz}, \delta \mathrm{ppm} / \mathrm{CDCl}_{3}\right): 176.5$ (C), $138.0(\mathrm{C}), 128.7(2 \mathrm{CH}), 128.1(\mathrm{CH}), 127.1(2 \mathrm{CH}), 69.1(\mathrm{CH}), 56.2$ (C), $35.8\left(\mathrm{CH}_{2}\right), 31.8\left(\mathrm{CH}_{3}\right), 30.7\left(\mathrm{CH}_{2}\right), 25.5\left(\mathrm{CH}_{2}\right), 25.5\left(\mathrm{CH}_{2}\right)$.

Spirobicyclic Pyrazolidinone $\mathbf{3 h}$ : To a solution of $\mathbf{9} \mathbf{h}_{\mathrm{Bn}}(510 \mathrm{mg}$, $1.42 \mathrm{mmol})$ in methanol $(100 \mathrm{~mL})$ was added $10 \% \mathrm{Pd} / \mathrm{C}(151 \mathrm{mg})$ and the solution was placed under an atmosphere of hydrogen with vigorous stirring for five days at room temperature. The resulting suspension was filtered through celite, concentrated in vacuo and purified by flash chromatography eluted with EtOAc/PE, 6:4 to afford pure $3 \mathrm{~h}(250 \mathrm{mg}, 78 \%)$ as a colorless oil. $R_{\mathrm{f}}(\mathrm{EtOAc} / \mathrm{PE}, 4: 6)=$ 0.20. HRMS (ESI+): $[\mathrm{M}+\mathrm{Na}]^{+}$calcd. for $\mathrm{C}_{11} \mathrm{H}_{18} \mathrm{~N}_{2} \mathrm{O}_{3} \mathrm{Na}^{+}$249.1210, found 249.1212. ${ }^{1} \mathrm{H}$ NMR $\left(300 \mathrm{MHz}, \delta \mathrm{ppm} / \mathrm{CDCl}_{3}\right): 4.79(\mathrm{~d}, J=$ $11.1 \mathrm{~Hz}, 1 \mathrm{H}), 4.25(\mathrm{qd}, \mathrm{J}=7.3,1.7 \mathrm{~Hz}, 2 \mathrm{H}), 4.03(\mathrm{~d}, J=11.1 \mathrm{~Hz}, 1 \mathrm{H})$, $3.05(\mathrm{~s}, 3 \mathrm{H}), 2.28-2.15(\mathrm{~m}, 1 \mathrm{H}), 1.89-1.64(\mathrm{~m}, 6 \mathrm{H}), 1.53-1.42(\mathrm{~m}, 1 \mathrm{H})$, $1.31(\mathrm{t}, \mathrm{J}=7.3 \mathrm{~Hz}, 3 \mathrm{H}) .{ }^{13} \mathrm{C} \mathrm{NMR}\left(75 \mathrm{MHz}, \delta \mathrm{ppm} / \mathrm{CDCl}_{3}\right): 175.6(\mathrm{C})$, $169.9(\mathrm{C}), 67.2(\mathrm{CH}), 61.7\left(\mathrm{CH}_{2}\right), 54.1(\mathrm{C}), 34.9\left(\mathrm{CH}_{2}\right), 31.9\left(\mathrm{CH}_{2}\right), 31.8$ $\left(\mathrm{CH}_{3}\right), 26.4\left(\mathrm{CH}_{2}\right), 26.0\left(\mathrm{CH}_{2}\right), 14.3\left(\mathrm{CH}_{3}\right)$.

Spirobicyclic Pyrazolidinone 3j: To a solution of $\mathbf{9 j}_{\mathrm{Bn}}(310 \mathrm{mg}$, $0.82 \mathrm{mmol})$ in methanol $(40 \mathrm{~mL})$ was added $10 \% \mathrm{Pd} / \mathrm{C}(88 \mathrm{mg})$ and the solution was placed under an atmosphere of hydrogen with vigorous stirring for three days at room temperature. The resulting suspension was filtered through celite, concentrated in vacuo and purified by flash chromatography eluted with EtOAc/PE, 6:4 to afford pure $3 \mathrm{~h}$ (134 mg, $67 \%)$ as a colorless oil. $R_{\mathrm{f}}(\mathrm{AcOEt} / \mathrm{PE}, 4: 6)=$ 0.10. HRMS (ESI+): $[\mathrm{M}+\mathrm{H}]^{+}$calcd. for $\mathrm{C}_{15} \mathrm{H}_{21} \mathrm{~N}_{2} \mathrm{O}^{+} 245.1648$ found 245.1647. ${ }^{1} \mathrm{H}$ NMR $\left(300 \mathrm{MHz}, \delta \mathrm{ppm} / \mathrm{CDCl}_{3}\right): 7.37-7.22(\mathrm{~m}, 5 \mathrm{H}), 4.14$ (broad s, 1H), $3.47(\mathrm{dd}, \mathrm{J}=9.7,2.5 \mathrm{~Hz}, 1 \mathrm{H}), 3.03(\mathrm{~s}, 3 \mathrm{H}), 2.87(\mathrm{dd}, \mathrm{J}=$ $14.0,2.5 \mathrm{~Hz}, 1 \mathrm{H}), 2.67(\mathrm{dd}, \mathrm{J}=14.0,10.6 \mathrm{~Hz}, 1 \mathrm{H}), 2.14-2.01(\mathrm{~m}, 1 \mathrm{H})$, 1.98-1.49 (m, 7H). ${ }^{13} \mathrm{C} \mathrm{NMR} \mathrm{(75} \mathrm{MHz,} \delta$ ppm/CDCl $): 177.6$ (C), 138.5 (C), $129.1(2 \mathrm{CH}), 128.6(2 \mathrm{CH}), 126.7(\mathrm{CH}), 66.4(\mathrm{CH}), 54.8(\mathrm{C}), 35.1$ $\left(\mathrm{CH}_{2}\right), 34.6\left(\mathrm{CH}_{2}\right), 31.8\left(\mathrm{CH}_{3}\right), 29.4\left(\mathrm{CH}_{2}\right), 26.2\left(\mathrm{CH}_{2}\right), 25.6\left(\mathrm{CH}_{2}\right)$.

Tetracyclic Pyrazolidinone 10a: To a solution of $\mathbf{1 0 a}_{\mathrm{Me}}(162 \mathrm{mg}$, $0.57 \mathrm{mmol})$ in anhydrous methanol $(6 \mathrm{~mL})$ was added LiOH $(14 \mathrm{mg}$, $0.58 \mathrm{mmol}$ ) and the resulting solution was stirred for five days at room temperature. Brine $(10 \mathrm{~mL})$ was then added and the aqueous layer was extracted three times with dichloromethane. The combined organic layers were dried with anhydrous sodium sulfate, fil- 
tered and concentrated in vacuo to give the crude product, which was purified by flash chromatography eluted with EtOAc/ $\mathrm{CH}_{2} \mathrm{Cl}_{2}$ 4:6 to afford pure 10 a $(47 \mathrm{mg}, 39 \%)$ as a yellow solid. Recrystallization of this material from dichloromethane gave monocrystalline prisms suitable for $\mathrm{X}$-ray diffraction analysis. ${ }^{[7]} R_{\mathrm{f}}(\mathrm{EtOAc} / \mathrm{DCM}, 4: 6)=$ 0.15. Mp 217.0-219.0 ${ }^{\circ} \mathrm{C}$. HRMS (ESI+): $[\mathrm{M}+\mathrm{H}]^{+}$calcd. for $\mathrm{C}_{14} \mathrm{H}_{15} \mathrm{~N}_{2} \mathrm{O}^{+}$ 227.1179, found 227.1177; $[\mathrm{M}+\mathrm{Na}]^{+}$calcd. for $\mathrm{C}_{14} \mathrm{H}_{14} \mathrm{~N}_{2} \mathrm{ONa}^{+}$ 249.0998, found 249.0996. ${ }^{1} \mathrm{H}$ NMR $\left(400 \mathrm{MHz}, \delta \mathrm{ppm} / \mathrm{CDCl}_{3}\right): 7.48$ (dd, $J=6.6,2.0 \mathrm{~Hz}, 1 \mathrm{H}), 7.43(\mathrm{dd}, J=6.3,2.5 \mathrm{~Hz}, 1 \mathrm{H}), 7.34-7.27(\mathrm{~m}$ 2H), $6.11(\mathrm{dd}, J=3.5,1.9 \mathrm{~Hz}, 1 \mathrm{H}), 4.96($ broad s, 1H), $4.52(\mathrm{~s}, 1 \mathrm{H})$ 3.39-3.26 (m, 1H), $2.94(\mathrm{~s}, 3 \mathrm{H}), 2.73(\mathrm{ddd}, \mathrm{J}=16.5,8.8,3.5 \mathrm{~Hz}, 1 \mathrm{H})$, $2.40(\mathrm{dd}, \mathrm{J}=12.2,6.6 \mathrm{~Hz}, 2 \mathrm{H}), 2.05(\mathrm{dd}, \mathrm{J}=12.2,9.3,9.3 \mathrm{~Hz}, 1 \mathrm{H})$. ${ }^{13} \mathrm{C}$ NMR $\left(75 \mathrm{MHz}, \delta \mathrm{ppm} / \mathrm{CDCl}_{3}\right): 173.7$ (C), 148.3 (C), 148.2 (C), $135.7(\mathrm{C}), 129.1(\mathrm{CH}), 128.6(\mathrm{CH}), 126.1(\mathrm{CH}), 122.1(\mathrm{CH}), 122.0(\mathrm{CH})$ $67.9(\mathrm{C}), 65.4(\mathrm{CH}), 37.5\left(\mathrm{CH}_{2}\right), 33.1\left(\mathrm{CH}_{2}\right), 31.9\left(\mathrm{CH}_{3}\right)$.

Tetracyclic Pyrazolidinone 1f: To a solution of $\mathbf{1 0 f}_{\mathrm{Bn}}(380 \mathrm{mg}$ $1.05 \mathrm{mmol})$ in methanol $(49 \mathrm{~mL})$ was added $10 \% \mathrm{Pd} / \mathrm{C}(104 \mathrm{mg})$ and the solution was placed under an atmosphere of hydrogen with vigorous stirring for four days at room temperature. The resulting suspension was filtered through celite, concentrated in vacuo and purified by flash chromatography eluted with EtOAc/PE, 6:4 to afford pure if $(230 \mathrm{mg}, 85 \%)$ as a colorless oil. $R_{\mathrm{f}}(\mathrm{AcOEt} / \mathrm{PE}, 4: 6)=$ 0.15. HRMS (ESI+): $[\mathrm{M}+\mathrm{H}]^{+}$calcd. for $\mathrm{C}_{15} \mathrm{H}_{19} \mathrm{~N}_{2} \mathrm{O}_{2}{ }^{+} 259.1441$, found 259.1442. It was not possible to obtain a well-resolved ${ }^{1} \mathrm{H}$ NMR spectrum at $294 \mathrm{~K}$. It was thus decided to analyze the corresponding trifluoromethanesulfonate salt $\mathbf{1 f} \cdot \mathbf{T f O H}$, which was prepared from a solution of $\mathbf{1 f}$ in dichloromethane and 1 equiv. triflic acid followed by evaporation of volatiles in vacuo. The amorphous solid obtained was recrystallized from ethyl acetate to give monocrystalline prisms suitable for $\mathrm{X}$-ray diffraction analysis. ${ }^{[7]}$ For the trifluoromethanesulfonate salt 1f.TfOH: Mp $238-239^{\circ} \mathrm{C}$. ${ }^{1} \mathrm{H}$ NMR $(300 \mathrm{MHz}$, $\left.\delta \mathrm{ppm} / \mathrm{C}_{6} \mathrm{D}_{6}\right): 7.77(\mathrm{~d}, J=8.5 \mathrm{~Hz}, 1 \mathrm{H}), 6.78(\mathrm{dd}, J=8.6,2.4 \mathrm{~Hz}, 1 \mathrm{H})$, $6.40($ broad s, 1H), $5.22(\mathrm{~s}, 1 \mathrm{H}), 3.79-3.68(\mathrm{~m}, 2 \mathrm{H}), 3.23(\mathrm{~s}, 3 \mathrm{H}), 3.12$ $(\mathrm{s}, 3 \mathrm{H}), 2.21-2.09(\mathrm{~m}, 1 \mathrm{H}), 1.88-1.70(\mathrm{~m}, 2 \mathrm{H}), 1.55-1.45(\mathrm{~m}, 1 \mathrm{H}), 1.39$ $1.26(\mathrm{~m}, 1 \mathrm{H}), 1.15-0.79(\mathrm{~m}, 2 \mathrm{H}) .{ }^{13} \mathrm{C}$ NMR $\left(75 \mathrm{MHz}, \delta \mathrm{ppm} / \mathrm{CDCl}_{3}\right)$ : $174.1(\mathrm{C}), 163.2(\mathrm{C}), 149.4(\mathrm{C}), 127.3(\mathrm{CH}), 125.6(\mathrm{C}), 115.5(\mathrm{CH}), 109.7$ $(\mathrm{CH}), 71.3(\mathrm{CH}), 61.1(\mathrm{C}), 56.6(\mathrm{CH}), 55.7\left(\mathrm{CH}_{3}\right), 36.2\left(\mathrm{CH}_{2}\right), 34.3\left(\mathrm{CH}_{2}\right)$, $31.4\left(\mathrm{CH}_{3}\right), 26.8\left(\mathrm{CH}_{2}\right)$. Resonance for $\mathrm{CF}_{3}$ not detected due to a low $\mathrm{S} / \mathrm{N}$ ratio.

\section{Acknowledgments}

Financial support from the Agence Nationale de la Recherche (ANR-13-JS07-0002-01), Aix Marseille Université, Centrale Marseille, and the Centre National de la Recherche Scientifique (CNRS) is gratefully acknowledged. We warmly thank Dr. Michel Giorgi (Aix-Marseille Université) for the X-ray structural analyses, Dr. Nicolas Vanthuyne (Aix-Marseille Université) and Ms. Marion Jean (CNRS) for analytical and semi-preparative HPLC on stationary phases, Dr. Jean-Valère Naubron (CNRS) for vibrational circular dichroism spectroscopy, and Prof. Dr. Cyril Bressy (AixMarseille Université) for advices with the synthesis of $\mathbf{3 b}$.

Keywords: Diastereoselectivity · C-H activation Organocatalysis - Spiro compounds . Synthetic methods

[1] a) Science of Synthesis Asymmetric Organocatalysis 1; Lewis Base and Acid Catalysts (Ed.: B. List), Thieme, Stuttgart, 2012; b) Science of Synthesis Asymmetric Organocatalysis 2: Brønsted Base and Acid Catalysts, and Additional Topics (Ed.: K. Maruoka), Thieme, Stuttgart, 2012; c) Comprehensive Enantioselective Organocatalysis (Ed.: P. I. Dalko), Wiley-VCH, Weinheim, 2013, Vol. 1-3.

[2] Seminal work and catalysts in Figure 1a: a) K. A. Ahrendt, C. J. Borths, D. W. C. MacMillan, J. Am. Chem. Soc. 2000, 122, 4243-4244; b) S. P. Brown, N. C. Goodwin, D. W. C. MacMillan, J. Am. Chem. Soc. 2003, 125, 1192-1194; C) M. Marigo, T. C. Wabnitz, D. Fielenbach, K. A. Jørgensen, Angew. Chem. Int. Ed. 2005, 44, 794-797; Angew. Chem. 2005, 117, 804; d) Y. Hayashi, H. Gotoh, T. Hayashi, M. Shoji, Angew. Chem. Int. Ed. 2005, 44, 4212-4215; Angew. Chem. 2005, 117, 4284. Reviews: e) A. Erkkilä, I. Majander, P. M. Pihko, Chem. Rev. 2007, 107, 5416-5470; f) M. Nielsen, D. Worgull, T. Zweifel, B. Gschwend, S. Bertelsen, K. A. Jørgensen, Chem. Commun. 2011, 47, 632-649; g) B. S. Donslund, T. K. Johansen, P. H. Poulsen, K. S. Halskov, K. A. Jørgensen, Angew. Chem. Int. Ed. 2015, 54, 13860-13874; Angew. Chem. 2015, 127, 14066; h) S. Meninno, C. Volpe, A. Lattanzi, ChemCatChem 2019, 11, DOI: https://doi.org/10.1002/ cctc. 201900569

[3] a) M. Lemay, W. W. Ogilvie, Org. Lett. 2005, 7, 4141-4144; b) M. Lemay, W. W. Ogilvie, J. Org. Chem. 2006, 71, 4663-4666; c) M. Lemay, L. Aumand, W. W. Ogilvie, Adv. Synth. Catal. 2007, 349, 441-447; d) H. He, B.-J. Pei, H.-H. Chou, T. Tian, W.-H. Chan, A. W. M. Lee, Org. Lett. 2008, 10, 2421-2424; e) Y. Langlois, A. Petit, P. Rémy, M.-C. Scherrmann, C. Kouklovsky, Tetrahedron Lett. 2008, 49, 5576-5579; f) E. Gould, T. Lebl, A. M. Z. Slawin, M. Reid, A. D. Smith, Tetrahedron 2010, 66, 8992-9008; g) F. Jakob, E. Herdtweck, T. Bach, Chem. Eur. J. 2010, 16, 7537-7546; h) I. Suzuki, M. Ando, R. Shimabara, A. Hirata, K. Takeda, Org. Biomol. Chem. 2011, 9, 3033-3040; i) E. Gould, T. Lebl, A. M. Z. Slawin, M. Reid, T. Davies, A. D. Smith, Org. Biomol. Chem. 2013, 11, 7877-7892; j) N. O. Häggman, B. Zank, H. Jun, D. Kaldre, J. L. Gleason, Eur. J. Org. Chem. 2018, 2018, 5412-5416.

[4] Reviews: a) W. Kirmse, Eur. J. Org. Chem. 2002, 2002, 2193-2256; b) Y. Coquerel, J. Rodriguez in Molecular Rearrangements in Organic Synthesis (Ed.: C. Rojas), Wiley, Hoboken, 2015, pp. 59-84.

[5] M. Presset, K. Mohanan, M. Hamann, Y. Coquerel, J. Rodriguez, Org. Lett. 2011, 13, 4124-4127.

[6] For precedents, see: a) M. C. Willis, C. K. Claverie, M. F. Mahon, Chem. Commun. 2002, 832-833; b) A. C. F. Cruz, N. D. Miller, M. C. Willis, Org. Lett. 2007, 9, 4391-4393; c) M. R. Albicker, N. Cramer, Angew. Chem. Int. Ed. 2009, 48, 9139-9142; Angew. Chem. 2009, 121, 9303; d) D. T. Ngoc, M. Albicker, L. Schneider, N. Cramer, Org. Biomol. Chem. 2010, 8, 17811784.

[7] CCDC 1922280 (for $\mathbf{1 f} \cdot \mathbf{C F}_{3} \mathbf{S O}_{3} \mathbf{H}$ ), and 1922281 (for 10a) contain the supplementary crystallographic data for this paper. These data can be obtained free of charge from The Cambridge Crystallographic Data Centre.

[8] S. Muthusamy, B. Gnanaprakasam, Tetrahedron Lett. 2005, 46, 635-638.

[9] J. B. Brazier, T. J. K. Gibbs, J. H. Rowley, L. Samulis, S. Chak Yau, A. R. Kennedy, J. A. Platts, N. C. O. Tomkinson, Org. Biomol. Chem. 2015, 13, 133-141. 